\title{
BMJ Open Effectiveness of implementing a best practice primary healthcare model for low back pain (BetterBack) compared with current routine care in the Swedish context: an internal pilot study informed protocol for an effectiveness- implementation hybrid type 2 trial
}

\author{
Allan Abbott, ${ }^{1}$ Karin Schröder, ${ }^{1}$ Paul Enthoven, ${ }^{1}$ Per Nilsen, ${ }^{2}$ Birgitta Öberg ${ }^{1}$
}

To cite: Abbott A, Schröder K, Enthoven $P$, et al. Effectiveness of implementing a best practice primary healthcare model for low back pain (BetterBack) compared with current routine care in the Swedish context: an internal pilot study informed protocol for an effectiveness-implementation hybrid type 2 trial. BMJ Open 2018;8:e019906. doi:10.1136/ bmjopen-2017-019906

- Prepublication history and additional material for this paper are available online. To view these files, please visit the journal online (http://dx.doi. org/10.1136/bmjopen-2017019906).

Received 2 0ctober 2017 Revised 28 February 2018 Accepted 5 March 2018

Check for updates

${ }^{1}$ Department of Medical and Health Sciences, Division of Physiotherapy, Faculty of Health Sciences, Linköping University, Linköping, Sweden

${ }^{2}$ Department of Medical and Health Sciences, Division of Community Medicine, Faculty of Health Sciences, Linköping University, Linköping, Sweden

Correspondence to

Dr Allan Abbott;

allan.abbott@liu.se

\section{ABSTRACT}

Introduction Low back pain (LBP) is a major health problem commonly requiring healthcare. In Sweden, there is a call from healthcare practitioners (HCPs) for the development, implementation and evaluation of a best practice primary healthcare model for LBP.

Aims (1) To improve and understand the mechanisms underlying changes in HCP confidence, attitudes and beliefs for providing best practice coherent primary healthcare for patients with LBP; (2) to improve and understand the mechanisms underlying illness beliefs, self-care enablement, pain, disability and quality of life in patients with LBP; and (3) to evaluate a multifaceted and sustained implementation strategy and the cost-effectiveness of the BetterBack $\odot$ model of care (MOC) for LBP from the perspective of the Swedish primary healthcare context. Methods This study is an effectiveness-implementation hybrid type 2 trial testing the hypothesised superiority of the BetterBack $\odot$ MOC compared with current routine care. The trial involves simultaneous testing of MOC effects at the HCP, patient and implementation process levels. This involves a prospective cohort study investigating implementation at the HCP level and a patient-blinded, pragmatic, cluster, randomised controlled trial with longitudinal follow-up at 3,6 and 12 months post baseline for effectiveness at the patient level. A parallel process and economic analysis from a healthcare sector perspective will also be performed. Patients will be allocated to routine care (control group) or the BetterBack $\odot$ MOC (intervention group) according to a stepped cluster dogleg structure with two assessments in routine care. Experimental conditions will be compared and causal mediation analysis investigated. Qualitative HCP and patient experiences of the BetterBack $*$ MOC will also be investigated. Dissemination The findings will be published in peer-reviewed journals and presented at national and international conferences. Further national dissemination and implementation in Sweden and associated national quality register data collection are potential future developments of the project.
Strengths and limitations of this study

- This will be the first study of effectiveness and implementation of a best practice model of care in low back pain primary care in Sweden.

- An international consensus framework is used for the development, implementation and evaluation of the BetterBack () model of care.

- The main trial's a priori methodology has been informed and refined by an internal pilot phase.

Date and version identifier 13 December 2017, protocol version 3.

Trial registration number NCT03147300; Pre-results.

\section{BACKGROUND}

Low back pain (LBP) is a prevalent and burdensome condition in Sweden and globally. ${ }^{2}$ LBP can be described by its location and by its intensity, duration, frequency and influence on activity. ${ }^{3}$ The natural course of LBP is often self-limiting, but a large majority experience pain recurrence and $20 \%$ may experience persistent symptoms. ${ }^{1}$ LBP is commonly categorised as non-specific, where a pathoanatomical cause cannot be confirmed through diagnostic assessment. ${ }^{4}$ Approximately $<1 \%-4 \%$ of LBP cases in primary healthcare may show signs underlying malignancy, fracture, infection or cauda equina syndrome requiring medical intervention. ${ }^{56}$ Furthermore, neuropathic pain may be present in $5 \%-15 \%$ of cases. ${ }^{78}$ Medical imaging studies display a high prevalence of varying spinal morphology and degenerative findings in 
both symptomatic and non-symptomatic younger and older adults. ${ }^{9}$ This suggests that LBP is more typically a result of benign biological and psychological dysfunctions, as well as social contextual factors influencing the pain experience.

In Sweden, previous studies by our research group suggest the healthcare process for patients with LBP tends to be fragmented, with many healthcare practitioners (HCPs) giving conflicting information and providing interventions of varying effectiveness. ${ }^{10} 11$ Our studies have shown that only a third of patients on sick leave for musculoskeletal disorders receive evidence-based rehabilitation interventions in primary care. ${ }^{10}{ }^{11}$ Furthermore our research has also demonstrated that there are still interventions that physiotherapists in primary care consider to be relevant in clinical practice despite the absence of evidence or consensus about the effects. ${ }^{12}$ Our preliminary data suggest that when patients with LBP are referred to specialist clinics, up to $48 \%$ have not received adequate evidence-based rehabilitation in primary care. There is therefore a strong case for change to address what care should be delivered for LBP and how to deliver it in the Swedish primary healthcare setting.

The development of best practice clinical guidelines aims to provide HCPs with recommendations based on strength of available evidence as well as professional consensus for the intervention's risk and benefits for the patients. Best practice clinical guidelines for LBP are lacking in Sweden but have recently been developed by the Danish Health and Medicines Authority and the English National Institute for Health and Care Excellence. ${ }^{13-15}$ These national guidelines provide a thorough assessment of current evidence and can be used in Sweden to form the basis for locally adapted recommendations. Common to LBP, central recommendations from best practice clinical guidelines for arthritis are also education and exercise therapy aimed at improving patient selfcare. Guideline-informed models of care (MOC) such as 'Better Management of Patients with Osteoarthritis (BOA)' in Sweden ${ }^{16}$ and 'Good Life with Osteoarthritis' in Denmark (GLA:D) ${ }^{17}$ have been successfully implemented with broad national HCP use. ${ }^{18} 19$ Furthermore, improvements in patient-reported pain, physical function and decreased use of pain medication after receiving these MOCs have been reported. ${ }^{18} 19$ A similar best practice MOC for LBP could potentially improve HCP evidencebased practice and patient-rated outcomes in the Swedish primary healthcare setting.

Recently an international consensus framework has been established to support the development, implementation and evaluation of musculoskeletal MOC. ${ }^{20} \mathrm{MOC}$ readiness for implementation requires that the MOC is informed by best practice recommendations, has a user focus and engagement, has a clear structure, and has a description of components as well as a description of how they are to be delivered. ${ }^{20}$ An important part of the MOC structure is the theoretical underpinning of how the
MOC intends to act on behavioural change mechanisms to attain specific behavioural targets. ${ }^{20}$ In order to achieve effective and efficient implementation of an MOC in primary healthcare, it is important to apply knowledge from implementation science. ${ }^{21-24}$ Implementation science is the scientific study of uptake of research findings and evidence-based practices into routine practice to improve the quality and effectiveness of healthcare and services. ${ }^{25}$ Implementation strategies focus on minimising barriers and maximising enablers that impact on the implementation and use of evidence-based practices. It has been suggested that a multifaceted strategy involving simultaneous use of several implementation strategies may be more effective than single-faceted strategies, but the evidence base is inconclusive. ${ }^{26} \mathrm{~A}$ recent systematic review however suggests that the most important aspects of successful implementation strategies are an increased frequency and duration of the implementation intervention and a sustained strategy. ${ }^{27}$

There is therefore a clear rationale for evaluating the extent to which and how a best practice MOC for LBP (BetterBack $\odot)$ ) implemented with a sustained multifaceted strategy is potentially effective in the Swedish primary care context. The costs in relation to effects are important to consider in order to deliver healthcare efficiently. This article describes a protocol for a BetterBack $\odot$ MOC effectiveness and implementation process evaluation. The protocol conforms to the Standard Protocol Items: Recommendations for Interventional Trials guidelines, ${ }^{28}$ with a checklist provided in online supplementary file 1 .

\section{AIMS}

The overall aim is to investigate the effectiveness and implementation process of the BetterBack (-) MOC for LBP in a Swedish primary healthcare context. The specific trial objectives are to (1) improve and understand the mechanisms underlying changes in HCP confidence, attitudes and beliefs for providing best practice primary healthcare for patients with LBP, (2) improve and understand the mechanisms underlying change in illness beliefs, self-care enablement, pain, disability and quality of life in patients with LBP, and (3) evaluate a multifaceted and sustained implementation strategy and cost-effectiveness of the BetterBack $\odot$ MOC for LBP in the Swedish primary healthcare context.

\section{HYPOTHESES}

1. HCP-reported confidence, attitudes and beliefs for providing primary healthcare for LBP will show statistically significant improvement after a sustained multifaceted implementation of the BetterBack $\odot$ MOC compared with baseline before implementation. Intentional and volitional HCP-rated determinants of implementation behaviour regarding the BetterBack $\odot$ MOC will mediate improved confidence, 
attitudes and beliefs in a causal effects model. This will correlate with more coherent care according to best practice recommendations.

2. The sustained multifaceted implementation of the BetterBack $\odot$ MOC will result in more statistically significant and greater clinically important improvement compared with current routine care for LBP regarding patient-reported measures for illness beliefs, self-care enablement, pain, disability and quality of life. Improvements in illness beliefs and adequate patient enablement of self-care will mediate the effect on these outcomes.

3. A sustained multifaceted implementation of the BetterBack $\odot$ MOC compared with current routine care will result in fewer patients with persisting LBP, fewer requiring specialist care, increased adherence to best practice recommendations and more statistically significant incremental cost-effectiveness ratio (ICER) based on cost per EuroQoL 5-Dimension Questionnaire (EQ-5D) quality-adjusted life years (QALY) gained.

\section{METHODS}

\section{Study design}

The WHO Trial Registration Data Set is presented in table 1. This study is an effectiveness-implementation hybrid type 2 trial testing the hypothesised superiority of the BetterBack $\odot$ MOC compared with current routine care. ${ }^{29}$ The design involves an effectiveness evaluation of the BetterBack $\odot$ MOC at the HCP and patient level, as well as a process evaluation of a sustained multifaceted implementation strategy conducted simultaneously. Evaluations are focused at the HCP and patient levels because the MOC is targeted at changing HCP behaviour, who then in turn implements behavioural change strategies at a patient level. This trial design was chosen for its potential to provide more valid effectiveness estimates based on pragmatic implementation conditions. This is in contrast to best or worst case implementation conditions common in traditional efficacy or effectiveness trials. ${ }^{29}$ Another advantage of the hybrid design is its potential to accelerate the translation of the MOC to real-world practice. This is in contrast to a time lag between efficacy, effectiveness and then dissemination steps in traditional research. ${ }^{29}$ The trial design is outlined in figure 1.

As outlined in table 2, the design at the HCP level involves data collection in the cohort before and prospectively after implementation of the BetterBack $\odot$ MOC. At the patient level, data are collected in a single-blinded, pragmatic, randomised controlled, stepped cluster format with longitudinal follow-up at 3, 6 and 12 months post baseline. Randomisation at the patient level is not possible due to potential carry-over effects of the HCP transitioning back and forth between providing routine care or the BetterBack $\odot$ MOC for different patients. Instead cluster randomisation is conducted at the start of the study, where patients are allocated thereafter to routine care (control group) or the BetterBack $\odot$ MOC (intervention group) depending on the clinic's allocation. Patients remain in their allocated group throughout the study.

A stepped cluster structure instead of a parallel structure of MOC implementation is applied due to the logistics involved in implementation in different geographical areas. The specific stepped cluster structure applied in the context of our study is classified as a dogleg with two assessments in routine care. ${ }^{30}{ }^{31}$ The term 'dog leg' has been used by methodologists because the stepped structure resembles the form of a dog hind leg. ${ }^{30}$ As displayed in table 2, this involves the first cluster being assessed after the implementation of the BetterBack $\odot$ MOC. The second cluster is assessed after a period of current routine care (control), and assessed again after the implementation of the BetterBack $\odot$ MOC. The third cluster receives current routine care (control) throughout the trial. However, studying the implementation of the BetterBack $\odot$ MOC in cluster 3 is planned to occur as a final step at the end of the study.

An advantage of using the dogleg structure with two assessments in routine care is that it allows for an internal pilot phase of initial implementation of the BetterBack (-) MOC in cluster 1 compared with clusters receiving current routine care. Another advantage is that data generated will still contribute to the final analyses to maintain trial efficiency. ${ }^{32}$ One objective for an internal pilot is to confirm the HCP acceptability of the intervention and trial within the first cluster. ${ }^{32}{ }^{33}$ A progression criteria for continuing the trial requires that HCPs who have completed the BetterBack $\odot$ education workshop rate on average a maximum of 2.5 out of 5 on the following determinants of implementation behaviour question: I expect that the application of BetterBack $\odot$ MOC will be useful $(1=$ agree completely to $5=$ do not agree at all $)$.

Another objective of the internal pilot is to monitor patient recruitment in all three clusters during the first 2 months to provide information on the optimal cross forward time for cluster 2. In the dogleg design it is possible to vary the time point of cluster 2 to cross forward from the control to intervention condition if the patient recruitment process in either cluster 1 or 3 is more or less than expected in the internal pilot (see table 2). In the event that cluster 1 recruits less than expected and cluster 2 or 3 recruits more than expected, then cluster 2 will cross forward to the intervention condition immediately after the internal pilot. If cluster 1 recruits more than expected and cluster 2 or 3 recruited less than expected during the internal pilot phase, then cluster 2 will cross forward to the intervention condition later in the trial to allow adequate current routine care data collection. Clusters were expected to recruit and gather data for at least 20 patients with LBP per month in the internal pilot. A final objective with the internal pilot phase is to assess baseline variation and change over 3 months for implementation process and patient primary outcome 


\section{Table 1 WHO Trial Registration Data Set}

\begin{tabular}{l}
\hline Data category \\
\hline Primary registry and trial identifying number \\
Date of registration in primary registry \\
Prospective registration \\
Secondary identifying numbers \\
Source(s) of monetary or material support \\
Secondary sponsor(s) \\
Contact for public queries \\
Contact for scientific queries \\
Public title \\
Scientific title
\end{tabular}

Countries of recruitment
Health condition(s) or problem(s) studied
Intervention(s)
Intervention(s)

Key inclusion and exclusion criteria

\section{Information}

ClinicalTrials.gov NCT03147300

3 May 2017

Yes

Not applicable

Linköping University

Linköping University

Not applicable

Allan Abbott, MPhysio, PhD (+46 (0)13 282 495) (allan.abbott@liu.SE)

Allan Abbott, MPhysio, PhD, Linköping University, Linköping, Sweden

Implementation of a best practice primary healthcare model for low back pain BetterBack $(-)$

Implementation of a best practice primary healthcare model for low back pain in Sweden

(BetterBack $\odot$ ): a cluster randomised trial

Sweden

\section{Low back pain}

Behavioural: current routine practice

Behavioural: multifaceted implementation of the BetterBack

Healthcare practitioner sample

Inclusion criteria

- Registered physiotherapists practising in the allocated clinics and regularly working with patients with low back pain.

Patient sample

Inclusion criteria

- Men and women 18-65years; fluent in Swedish; accessing public primary care due to a current episode of a first-time or recurrent debut of benign low back pain with or without radiculopathy.

Exclusion criteria

- Current diagnosis of malignancy, spinal fracture, infection, cauda equina syndrome, ankylosing spondylitis or systemic rheumatic disease, previous malignancy during the past 5 years; current pregnancy or previous pregnancy up to 3 months before consideration of inclusion; patients who fulfil the criteria for multimodal/multiprofessional rehabilitation for complex long-standing pain; severe psychiatric diagnosis.

\section{Study type}

Interventional

Date of first enrolment

1 April 2017

Target sample size

600

Recruitment status

\section{Recruiting}

Primary outcome(s)

- Incidence of participating patients receiving specialist care (time frame: 12 months after baseline)

- Numeric Rating Scale for lower back-related pain intensity during the latest week (time frame: change between baseline and 3 months post baseline)

- Oswestry Disability Index V.2.1 (time frame: change between baseline and 3 months post baseline)

- Practitioner Confidence Scale (time frame: change between baseline and 3 months post baseline)

Key secondary outcomes
- Clinician-rated healthcare process measures (time frame: baseline and final clinical contact (up to 3 months where the time point is variable depending on the amount of clinical contact required for each patient))

- Numeric Rating Scale for lower back-related pain intensity during the latest week (time frame: baseline, 3, 6 and 12 months)

- Oswestry Disability Index V.2.1 (time frame: baseline, 3, 6 and 12 months)

- Pain Attitudes and Beliefs Scale for physical therapists (time frame: baseline, directly after education and at 3 and 12 months afterwards)

- Patient Enablement Index (time frame: 3, 6 and 12 months)

- Patient Global Rating of Change (time frame: 3, 6 and 12 months)

- Patient Satisfaction (time frame: 3, 6 and 12 months)

- Practitioner Confidence Scale (time frame: baseline, directly after commencement of implementation strategy and at 3 and 12 months afterwards)

- The Brief Illness Perception Questionnaire (time frame: baseline, 3, 6 and 12 months)

- The European Quality of Life Questionnaire (EuroQoL 5-Dimension Questionnaire) (time frame: baseline, 3, 6 and 12 months 


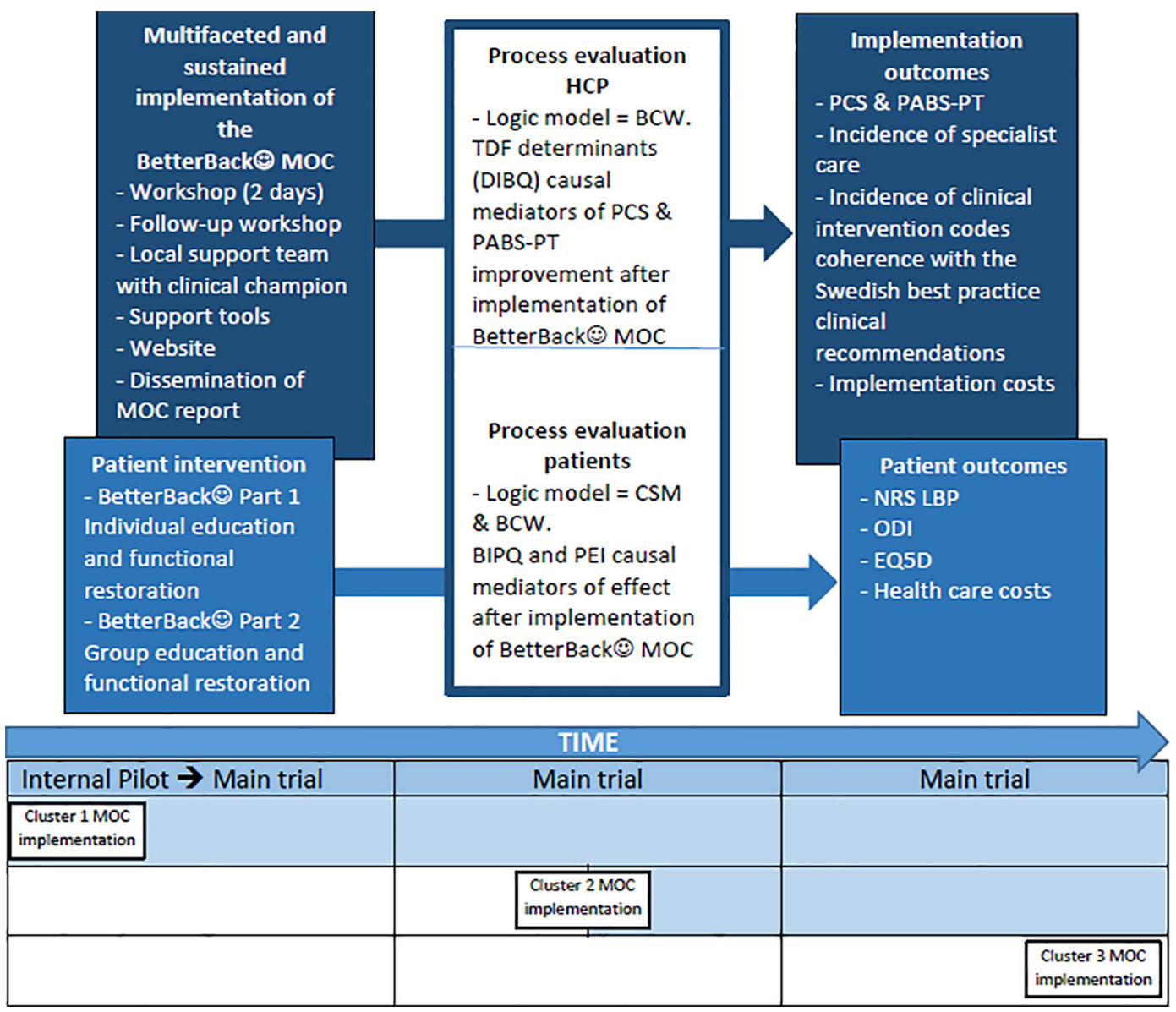

Figure 1 Effectiveness-implementation hybrid type 2 trial design with chronological sequence of intervention in each cluster. BCW, Behaviour Change Wheel; BIPQ, Brief Illness Perception Questionnaire; CSM, Common Sense Model of Self-Regulation; DIBQ, Determinants of Implementation Behaviour Questionnaire; EQ-5D, EuroQoL 5-Dimension Questionnaire; HCP, healthcare practitioner; MOC, model of care; NRS LBP, Numeric Rating Scale for lower back-related pain; ODI, Oswestry Disability Index; PABS-PT, Pain Attitudes and Beliefs Scale for physical therapists; PCS, Practitioner Confidence Scale; PEI, Patient Enablement Index; TDF, Theoretical Domains Framework.

measures to inform if our a priori sample size calculation needed to be revised in the continuation of the trial.

\section{Study setting}

The Östergötland public healthcare region has a total population of 453596 inhabitants with approximately 5000 patients per year accessing primary care physiotherapy due to LBP. In the public healthcare region of Östergötland, a large majority of consultations for LBP are via direct access to the 15 primary care physiotherapy rehabilitation clinics. A smaller percentage of consultations are via referral to these rehabilitation clinics from the 36 primary healthcare general practices in the region. Therefore the focus of this study is on the physiotherapeutic rehabilitation process for LBP in primary care. The rehabilitation clinics form three clusters in Östergötland healthcare region. These clusters are based on municipal geographical area and organisational structure of the rehabilitation clinics, which help to minimise contamination between separate clusters of clinics (figure 2). Cluster west comprised 5 clinics with 27 physiotherapists, cluster central comprised 6 clinics with 44 physiotherapists, and cluster east comprised 6 clinics with 41 physiotherapists.

\section{Eligibility criteria}

Registered physiotherapists practising in the allocated clinics and regularly working with patients with LBP will be included in the study. These physiotherapists will assess the eligibility of consecutive patients before and after the implementation of the BetterBack $\odot$ MOC based on the following criteria:

- Inclusion criteria: men and women 18-65 years; fluent in Swedish; and accessing public primary care due to a first-time or recurrent episode of acute, subacute or chronic-phase benign LBP with or without radiculopathy.

- Exclusion criteria: current diagnosis of malignancy, spinal fracture, infection, cauda equina syndrome, ankylosing spondylitis or systemic rheumatic disease, previous malignancy during the past 5 years; spinal surgery during the last 2 years; current pregnancy or previous pregnancy up to 3 months before consideration of inclusion; patients who fulfil the criteria for multimodal/multiprofessional rehabilitation for complex long-standing pain; and severe psychiatric diagnosis. 


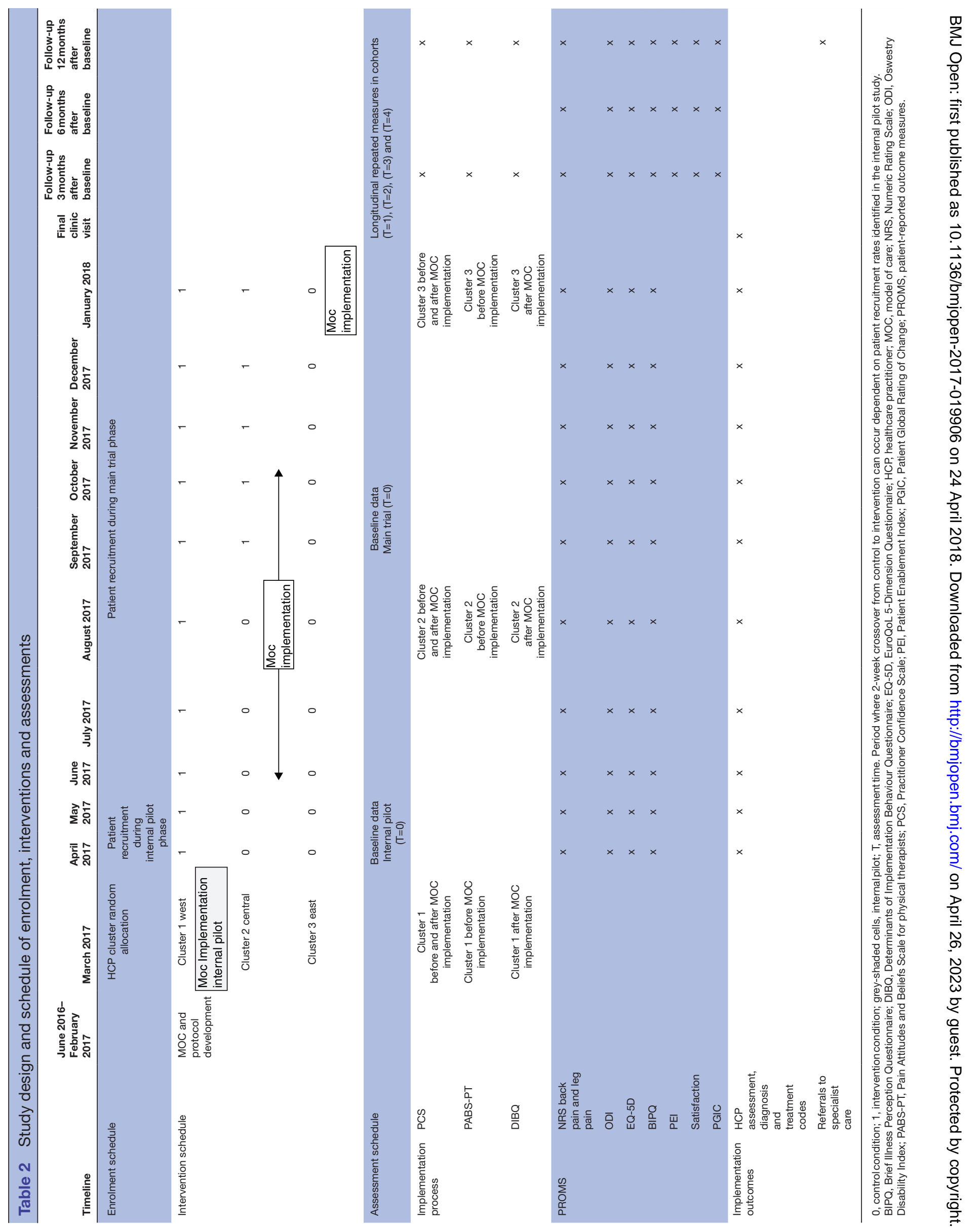




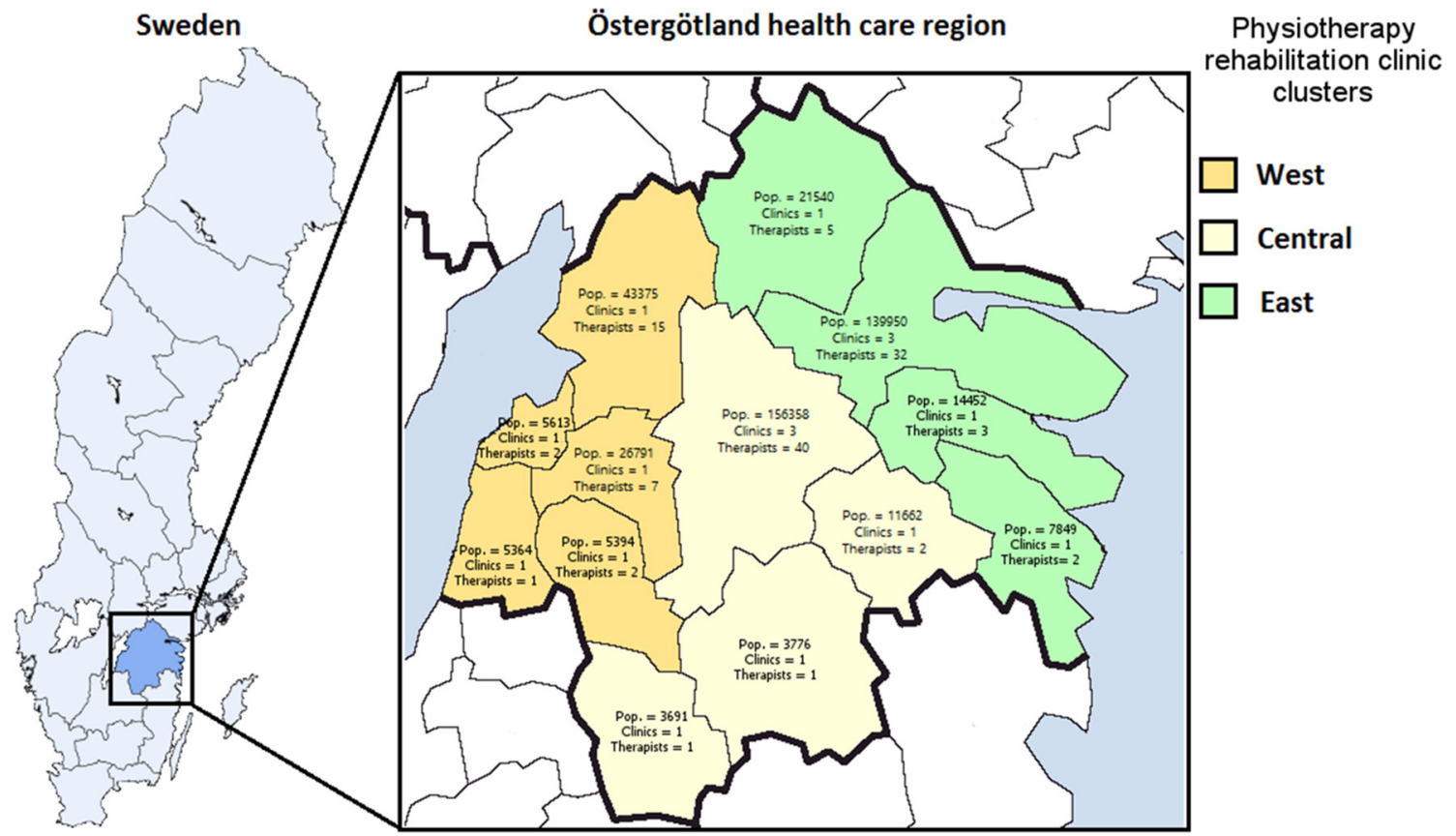

Figure 2 Municipal resident population and number of physiotherapy rehabilitation clinics and therapists in the west, central and east organisational clusters in Östergötland healthcare region.

\section{Interventions}

Control condition: current routine physiotherapeutic care for LBP in primary healthcare

Patients attending rehabilitation clinic clusters that have not yet completed the implementation of the BetterBack $\odot$ MOC will receive treatment as usual according to current routine care clinical pathways (figure 3). A clinical pathway specified in Östergötland public healthcare region requires that for patients accessing primary care due to LBP, a triage is to be performed by licensed HCPs (physiotherapists, nurses or general practitioners (GPs)), to triage for specific pathology of serious nature. These approximately $1 \%-4 \%$ of patients with suspected specific pathology of serious nature are then to be examined by GPs and referred for specific intervention in secondary or tertiary healthcare. The majority of patients with LBP who on initial triage are assessed as having benign LBP are then scheduled for physiotherapy consultation and implementation of an LBP management plan. If the patient has persistent functional impairment and activity limitation despite 2-3 months of primary care intervention, the clinical pathway specifies inclusion criteria for specialist care referral pathways (figure 3).

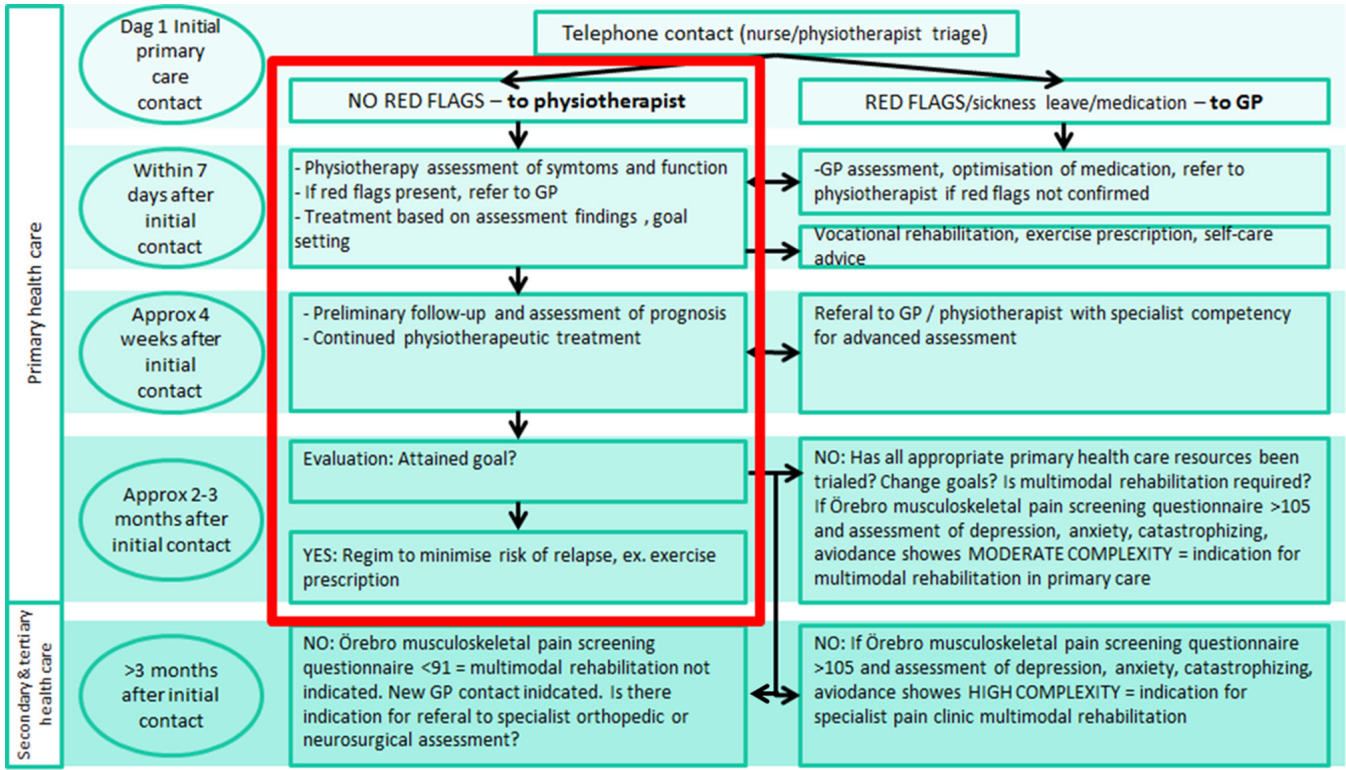

Figure 3 Current routine care clinical pathway for LBP in Östergötland healthcare region. The primary care physiotherapy process outlined by the red square is the focus area for the implementation of the BetterBack $\odot$ model of care for LBP. GP, general practitioner; LBP, low back pain. 
Intervention condition: the BetterBack $\odot$ MOC for LBP Development, design and implementation of the BetterBack $\odot$ $M O C$ for $\angle B P$

A framework for the development of musculoskeletal $\mathrm{MOC}^{20}$ was used to guide the development of the BetterBack $\odot$ MOC for LBP. The high prevalence and burden of LBP, ${ }^{12}$ discordance in evidence-based rehabilitation processes, ${ }^{10-12}$ a lack of clinical practice guidelines and a call for a best practice MOC requested by physiotherapy clinic managers in the Östergötland healthcare region have been identified in the primary care of LBP. Therefore, a case for change has been justified to improve current physiotherapeutic health service delivery for the primary care of LBP. The content and structure of the BetterBack $\odot$ MOC were developed by engaging a work group of physiotherapy clinicians (clinical champions) from each primary care cluster in the Östergötland public healthcare region and physiotherapy academics at Linköping University. A Template for Intervention Description and Replication checklist ${ }^{34}$ is described in online supplementary file 2. To identify which key areas of contemporary care were of relevance for the BetterBack $\odot$ MOC, the following tasks were performed by the work group:

1. Discussion and outline of the current routine care clinical pathway for LBP and areas needing improvement: the work group concluded that the BetterBack $\odot$ MOC needed to focus on the following:

- WHO/WHERE: the primary care physiotherapy process for the management of patients with LBP in Östergötland healthcare region outlined by the red square in figure 3.

2. Analysis and discussion of existing international best practice clinical guidelines: the following thorough and up-to-date systematic critical literature reviews and international clinical guidelines were analysed and discussed by the work group: refs 13-15 35 .

3. Adaptation of best practice clinical guidelines to the Swedish context: the development of evidence-based recommendations was based on the Swedish National Board of Health and Welfare methods for guideline construction. ${ }^{36}$ The overall grade of evidence together with a consensus position based on professional experience and patient net benefit versus harms and costs are the key aspects on which the work group has formulated local recommendations to reflect their strength. ${ }^{37}$ The recommendations have been externally reviewed by local physicians and international experts from the University of Southern Denmark. A summary of the Östergötland healthcare region physiotherapeutic clinical practice guideline recommendations for primary care management of LBP with or without radiculopathy as well as the support tools used in the BetterBack $\odot$ MOC is provided in online supplementary file 3 .

4. Considering potential barriers to the uptake of evidence-based recommendations by $\mathrm{HCP}^{38}$ the work group identified and discussed targeted HCP behavioural change priorities of relevance for the BetterBack - .
MOC. The work group discussion led to a rationale for the BetterBack $\odot$ MOC content and implementation described in table 3:

- WHY: The main HCP target behaviour was the adoption of the BetterBack $\odot$ MOC to influence HCP delivery of care coherent with best practice recommendations.

- WHAT: This would require the contents of the MOC to change impeding barrier behaviours such as low confidence in skills/capabilities for improving LBP patient management, a biomedical treatment orientation rather than a biopsychosocial orientation, and low awareness or beliefs of the negative consequences of the MOC. ${ }^{38}$

- HOW: BetterBack $\odot$ MOC content used to overcome the modifiable barriers includes support tools aimed at further education and enablement of HCP clinical reasoning in providing LBP assessment and treatment coherent with the Swedish adaptation of best practice clinical guidelines. The support tools include assessment proformas with associated instruction manual, clinical reasoning flow charts linking assessment findings to relevant treatment interventions, patient education brochures and group education material on LBP self-care, as well as a functional restoration programme (online supplementary file 3 ).

- WHEN/HOW MUCH/TAILORING: The functional restoration programme and patient education components used, and their individual and groupbased delivery and dosing, are individualised based on the HCP clinical reasoning of the type and grade of patients' functional impairments and activity limitations (online supplementary file 3 ).

- PROCEDURE: Figure 4 displays a flow diagram showing the steps involved for HCPs in delivering the contents of the BetterBack (-) MOC.

The Behaviour Change Wheel (BCW) ${ }^{39}$ was used by the work group as a logic model to theorise the process of how the BetterBack $\odot$ MOC content applied at the guideline policy level could guide theory-informed intervention functions using specific behavioural change techniques. ${ }^{40}$ To help investigate possible mediators of behavioural change interventions in the BetterBack $\odot$ MOC, the Theoretical Domains Framework (TDF) ${ }^{41}$ was integrated into the BCW. The TDF comprised 14 theoretical domains/ determinants of behavioural change which could potentially influence behavioural change technique effect on the central source of behaviour. ${ }^{42}$ The central source of behaviour in the behavioural change wheel is described by the COM-B model. In the COM-B model, a person's capability (physical and psychological) and opportunity (social and physical) can influence on motivation (automatic and reflective), enacting behaviours that can then alter capability, motivation and opportunity. ${ }^{39}$ The $\mathrm{BCW}^{39}$ and $\mathrm{TDF}^{41}$ are displayed in figure 5 .

5. The following sustained multifaceted implementation strategy for the BetterBack $\odot$ MOC was developed: 


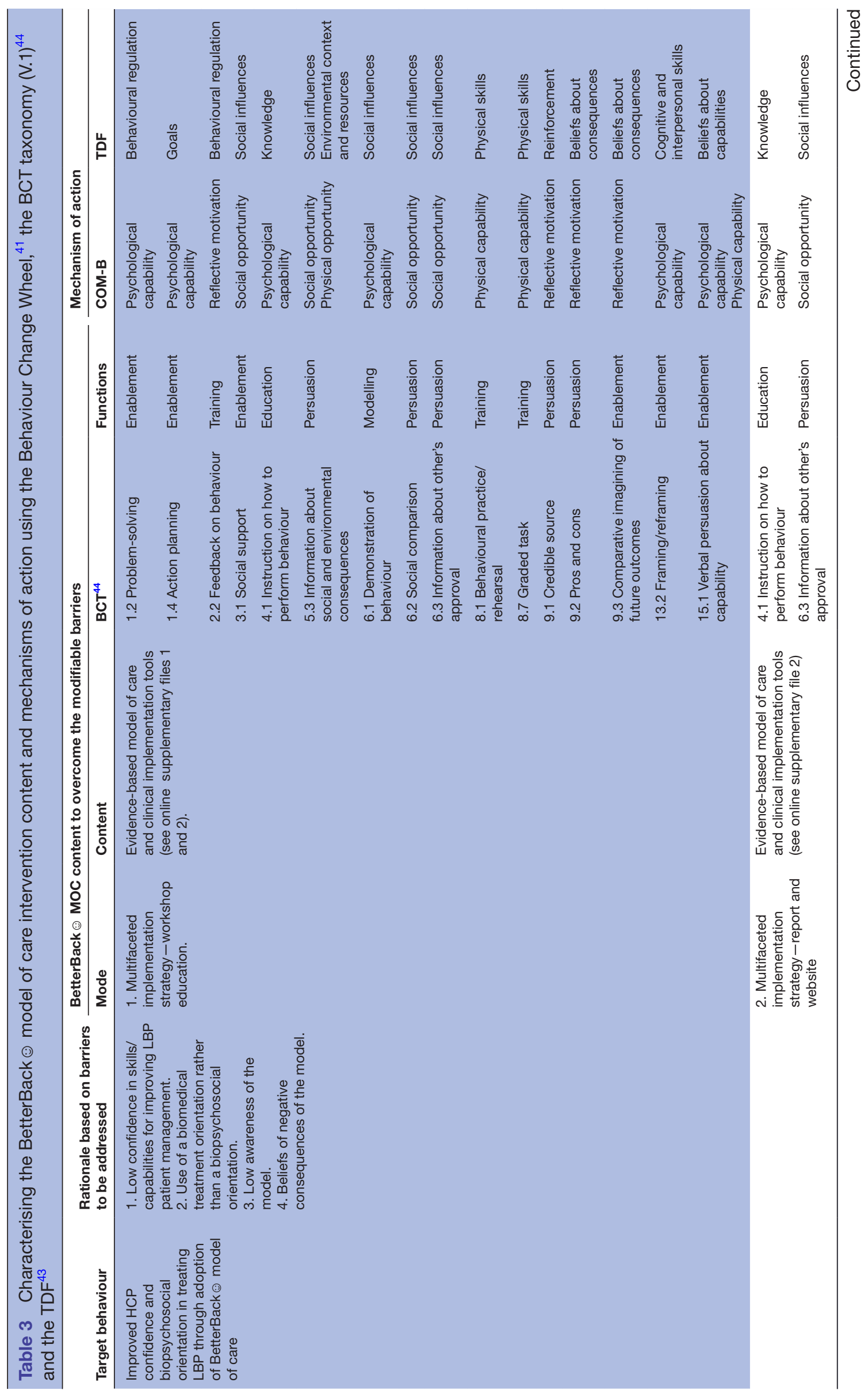




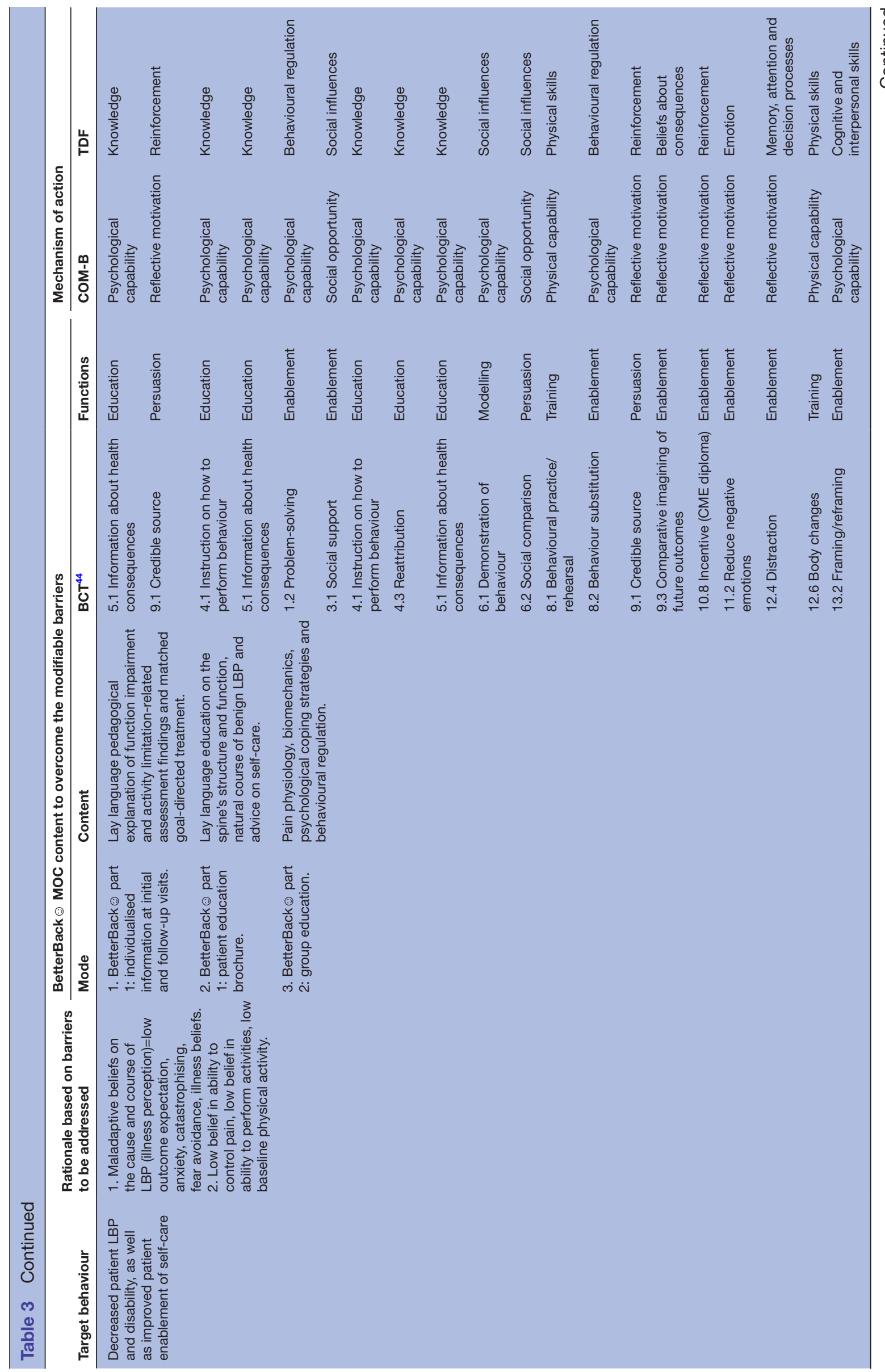

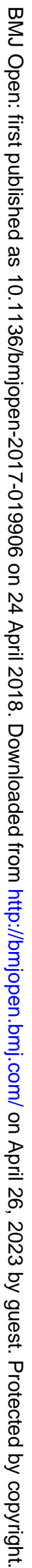




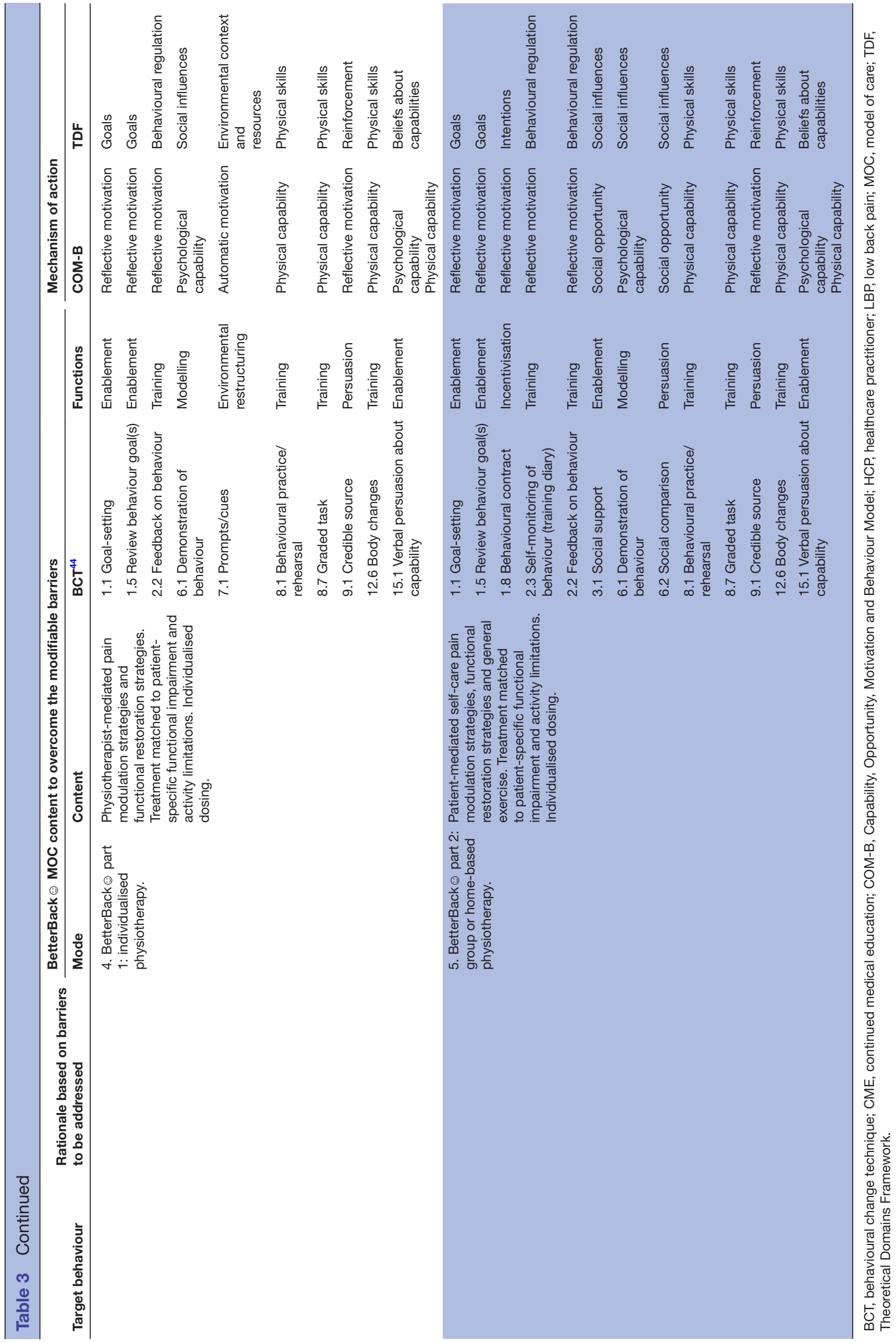




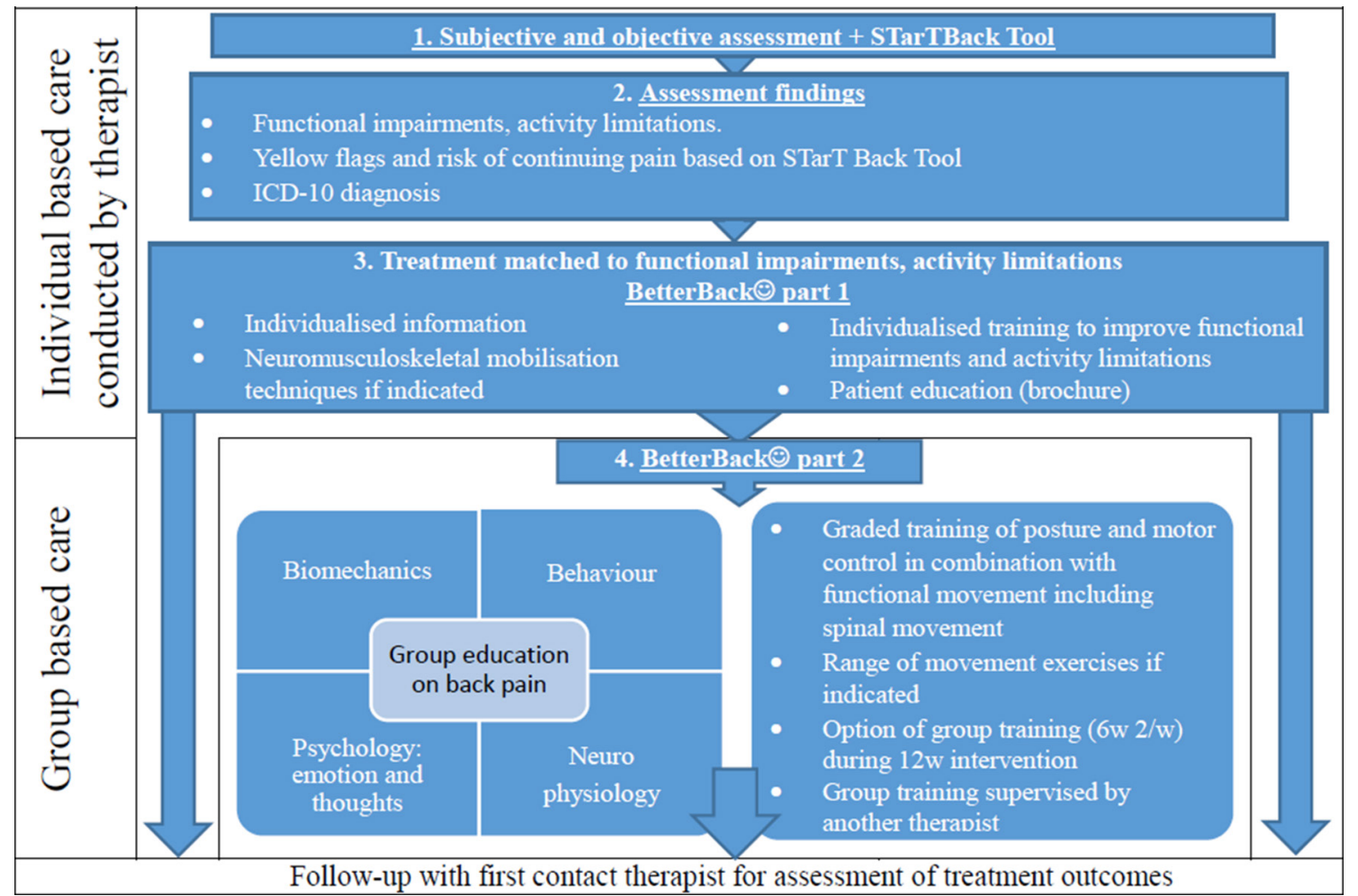

Figure 4 Steps involved for healthcare practitioners in delivering the contents of the BetterBack $\odot$ model of care. ICD-10, International Classification of Diseases-10.

- An implementation forum including rehabilitation unit managers and clinical researchers was formed. The implementation forum collaborated on forming overarching goals, timeline and logistics facilitating and sustaining the implementation of the BetterBack $\odot$ MOC in the primary care rehabilitation clinic clusters in the Östergötland public healthcare region.

- An MOC support team was formed. This comprised experienced clinicians (clinical champions) from each rehabilitation unit together with clinical researchers facilitating local implementation and sustainability of the BetterBack $\odot$ MOC at the rehabilitation units.

- A package of education and training that the support team can use to assist the use of the BetterBack $\odot$ MOC by HCP was developed.

- Physiotherapists in the three geographical clusters of public primary care rehabilitation clinics in Östergötland will be offered to participate in a 13.5-hour (2 days) continued medical education workshop. The workshop is designed by the

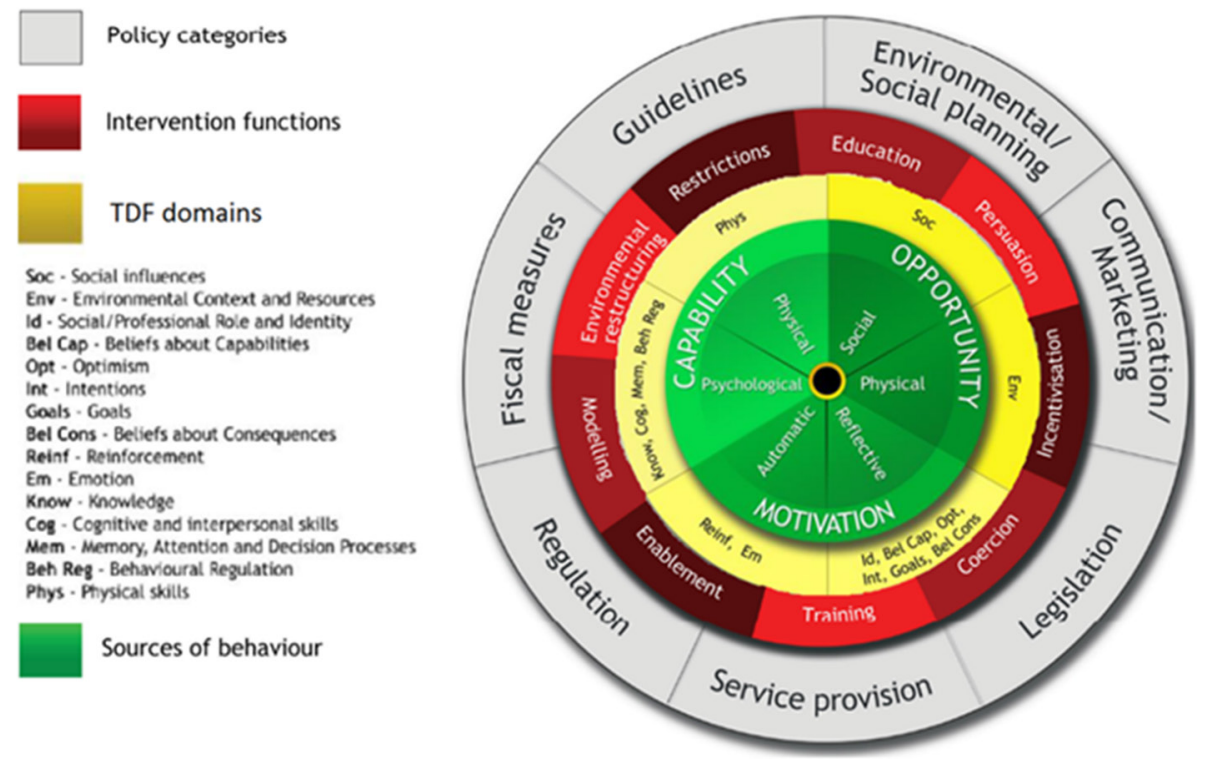

Figure 5 The Behavioural Change Whee ${ }^{39}$ and the Theoretical Domains Framework (TDF). ${ }^{41}$ 
support team with at least two clinical researchers and one experienced clinician from the rehabilitation unit cluster present in the support team's delivery of the workshop for each cluster. The HCP education provided in the workshop format is described in online supplementary file 4 .

- Key components of the educational programme are the following:

- Education and persuasion about evidence-based recommendations for LBP care and the BetterBack $\odot$ MOC through an experiential learning process applying problem-based case studies and clinical reasoning tools.

- Training and modelling of the practical use of the BetterBack $\odot$ education and physical intervention programmes aiming at self-care, as well as function and activity restoration.

- Access to a website describing the BetterBack $\odot$ MOC. A chat forum will give an opportunity for clinicians to ask questions and share different experiences of the new strategy managed by the support team. Researchers will respond to questions from the participating clinicians.

- To consolidate the BetterBack $\odot$ MOC use at the local clinics, the local support team member and clinical researchers will mediate a 2-hour interactive follow-up workshop 3 months after BetterBack $\odot$ MOC implementation. Aspects of the previous workshop content will be discussed and reinforced. To aid continued sustainability of the BetterBack $\odot$ MOC implementation, the local support team member will provide continued maintenance of education at their clinics and even educate new staff.

6. Once HCP behaviour change has occurred, it is anticipated that HCP use of the BetterBack $\odot$ MOC may influence patient outcomes. A rationale for causal mediation effects can be proposed based on the Common Sense Model of Self-Regulation. ${ }^{42}$ This suggests a potential effect of the BetterBack $\odot$ MOC on improved patient-reported pain, physical function and quality of life may be mediated by improved patient illness beliefs, such as cognitive and emotional illness representations, as well as adequate coping through self-care enablement. ${ }^{42}$ The patient target behaviours are therefore focused on the understanding of the mechanisms and natural course of benign LBP and the enablement of self-care. This requires content of the MOC to change patients' impeding barrier behaviours such as maladaptive illness beliefs on the cause and persistent course of LBP (low outcome expectation, anxiety, catastrophising, fear avoidance and negative illness beliefs), low self-care enablement and low baseline physical activity. ${ }^{43}$ The content for the patient education and functional restoration programme included in the BetterBack $\odot$ MOC therefore reflects these aspects and is shown in online supplementary file 3 . These are also characterised according to the BCW, behavioural change technique taxonomy ${ }^{44}$ and TDF in table 3.

\section{OUTCOMES}

\section{Implementation process}

\section{Primary outcome measure}

- Practitioner Confidence Scale (PCS $)^{45}$ mean change from baseline to 3 months post baseline. Practitioner-reported confidence is the primary HCP behavioural change goal for the HCP education and training workshop in the multifaceted implementation of the BetterBack $\odot$ MOC. The 3-month time frame allows for the development and consolidation of HCP behavioural change after application in repeated patient cases.

\section{Secondary outcome measures}

- $\mathrm{PCS}^{45}$ mean immediate change from baseline to directly after the HCP education and training workshop, as well as mean long-term change from baseline to 12 months post baseline. This secondary outcome is important for the understanding of longitudinal HCP behavioural change.

- Pain Attitudes and Beliefs Scale for physical therapists (PABS-PT $)^{46}$ mean change from baseline to directly after the HCP education and training workshop, as well as at 3 and 12 months post baseline.

\section{Implementation outcomes}

Primary outcome measure

- Proportional difference between control and intervention groups for incidence of participating patients receiving specialist care for LBP between baseline and 12 months after baseline. Incidence proportion, analogous to cumulative incidence or risk, is calculated by taking the number of patients receiving specialist care of LBP and dividing it by the total number of patients recruited to the study. The main goal of both the control and intervention conditions in primary care for benign first-time or recurrent debut of LBP is to improve patient-reported outcomes without the need of secondary or tertiary healthcare processes.

Secondary outcomes measures

- Mean difference between control and intervention groups for change between baseline and final clinical visit regarding grade of patients' functional impairment and activity limitation according to the International Classification of functioning, disability and health (ICF) brief core set for LBP. ${ }^{47}$

- The proportion of patients who receive the BetterBack $\odot$ MOC and registration of healthcare codes coherent with the Swedish best practice clinical recommendations.

\section{Patient outcomes}

Primary outcome measure

- Numeric Rating Scale for lower back-related pain intensity (NRS-LBP) during the latest week. ${ }^{48}$ The mean difference between control and intervention groups in change between baseline and 3 months post 
baseline will be analysed. Pain intensity is the primary functional impairment that patients with LBP contact primary healthcare for and has been recommended by international consensus to be included as a core outcome domain for clinical trials in non-specific $\mathrm{LBP}^{49}$ International consensus even recommends patient-reported NRS change over 6 months as a core metric for pain management interventions. ${ }^{50}$

- Oswestry Disability Index V.2.1 (ODI). ${ }^{51}$ The mean difference between control and intervention groups in change between baseline and 6 months post baseline will be analysed. Disability, analogous to decreased physical functioning and activity limitation, has been recommended by international consensus to be included as a core outcome domain for clinical trials in non-specific LBP. ${ }^{49}$ International consensus even recommends patient-reported ODI change over 6 months as a core metric for functional restoration. ${ }^{50}$

\section{Secondary outcome measures}

- NRS-LBP ${ }^{48}$ and ODI $^{50}$ mean difference between control and intervention groups in short-term change from baseline to 3 months post baseline and mean long-term change from baseline to 12 months post baseline. These secondary outcomes are important for the understanding of longitudinal patient-rated changes in pain intensity and disability after primary care intervention.

- The European Quality of Life Questionnaire (EQ-5D) ${ }^{52}$ The mean difference between control and intervention groups in change between baseline and 3, 6 and 12 months post baseline will be analysed. Health-related quality of life has been recommended by international consensus to be included as a core outcome domain for clinical trials in non-specific LBP. $^{49}$ International consensus even recommends patient-reported EQ-5D change over 6 months as a core metric for pain management interventions. ${ }^{50}$

- The Brief Illness Perception Questionnaire (BIPQ) ${ }^{53}$ The mean difference between control and intervention groups in change between baseline and 3, 6 and 12 months post baseline will be analysed. Illness perception has been shown to predict longitudinal pain and disability outcomes in several LBP studies. ${ }^{54-58}$

- Patient Enablement Index (PEI) ${ }^{59}$ Patient Global Rating of Change (PGIC) ${ }^{60}$ and Patient Satisfaction $(\mathrm{PS})^{61}$ mean difference between control and intervention groups at 3, 6 and 12 months post baseline will be analysed.

\section{Participant timeline}

The trial timeline is shown in table 2. The intervention schedule started with the development of evidencebased recommendations and the BetterBack $\odot$ MOC, which occurred during June 2016-February 2017. The enrolment schedule started with cluster enrolment and randomisation in March 2017. This resulted in the first allocated cluster 1 (west) entering internal pilot of implementing the BetterBack $\odot$ MOC HCP education and training workshop, which occurred in March 2017. This was followed up with a 2-month internal pilot of patient enrolment schedule occurring in all three clusters during April-May 2017. In order to finalise a sample size calculation for the main trial, baseline data collected during the internal pilot are compared with follow-up data 3 months after baseline for the primary outcome measure questionnaires to analyse initial HCP and patient effects of the implementation of BetterBack $\odot$ MOC in cluster 1 compared with the control conditions in clusters 2 and 3 . In the transition to the main trial, patient enrolment and baseline assessments will then continue to occur until January 2018. The eventual time of crossing forward of cluster 2 into the implementation of the BetterBack $\odot$ MOC is determined by the internal pilot trial results. Participants in the trial will be followed up longitudinally at 3, 6 and 12 months after baseline measures. The schedule for assessments is also outlined in table 2.

\section{Sample size}

An initial sample size estimation in the planning stage of the study assumed at least a small Cohen's d effect size $(d=0.35)$ for the HCP behavioural change primary and secondary outcomes. This is based on previous literature showing small-moderate HCP behavioural change effects sizes using similar interventions to increase the uptake of evidence-based management of LBP in primary care. ${ }^{62} 63$ Considering also a one-tailed $\mathrm{P}=0.05$ for the benefit of the multifaceted implementation of the BetterBack $\odot$ MOC, an $80 \%$ statistical power and a $20 \%$ loss to follow-up, a sample size of $n=63 \mathrm{HCP}$ is needed for a matched pairs t-test statistics comparing baseline and follow-up means. We assume a possible carry-over of a similar effect size $(\mathrm{d}=0.35)$ on patient behavioural change primary and secondary outcomes. Considering also a one-tailed $\mathrm{P}=0.05$ for the benefit of the multifaceted implementation of BetterBack $\odot$ MOC compared with usual care and an $80 \%$ statistical power, the number of patients required for an individually randomised simple parallel group design would be $n=204$. Adjusting for the design effect due to cluster randomising, an intracluster correlation of 0.01 and a cluster autocorrelation of 0.80 , a dogleg design with two assessments in routine care and 100 patients in each cluster section would require at least $n=402$ patients over 2.41 clusters according to algorithms described by Hooper and Bourke. ${ }^{30}$ In a balanced recruitment schedule, this equates to 14 patients per month per cluster for a total of 3 clusters. Allowing for potential unbalanced recruitment flow and a potential dropout in the longitudinal outcomes at 3, 6 and 12 months post baseline, each cluster will aim for up to 20 patients per month, equating to a potential total study of $n=600$.

\section{Recruitment}

In an effort to curb recruitment difficulties, strategies to promote adequate enrolment of participants into the study will be used. We anticipate less problems with 
recruitment into the prospective cohort study design investigating the multifaceted implementation of the BetterBack $\odot$ MOC at the HCP level. This is due to the study having been endorsed by clinical department managers calling all HCPs working with patients with LBP at their clinics to participate. However, recruitment of patients into the cluster randomised controlled trial is dependent on the feasibility of recruitment processes adapted to the context of each individual clinic and the compliance of HCPs to administer recruitment of consecutive patients. A strategy to optimise the administration of patient recruitment will involve the author KS regularly visiting participating clinics to inform HCPs of the study protocol and help streamline practical administration of the protocol in the context of the individual clinics. KS will also monitor weekly recruitment rates from the clinics and provide motivational feedback on recruitment flow to clinical department managers and designated clinical champions who will provide additional motivational feedback to HCP. In accordance with the Consolidated Standards of Reporting Trials, a flow diagram displaying participant enrolment, allocation, follow-up and analysis will be constructed. ${ }^{64}$ Reasons for exclusion, declined participation, protocol violations and loss to follow-up will be monitored by KS.

\section{Allocation and blinding}

Random concealed allocation of clusters was performed by a blinded researcher randomly selecting from three sequentially numbered, opaque, sealed envelopes. The method resulted in the following order: $1=$ clusterwest, $2=$ cluster central and $3=$ cluster east. KS informed the clinics in the different clusters of their allocation to the control or intervention study condition. Due to the nature of the study and intervention, HCPs conducting patient measurements and treatment cannot be blinded to group allocation. Risk of bias is minimal as the primary and secondary outcomes are patient self-reported questionnaires. Patients will be blinded to group allocation. The researcher responsible for statistical analysis will not be blinded to group allocation, but an independent statistician will review statistical analysis.

\section{Data collection}

Data will be collected through quantitative questionnaires and qualitative focus group and semistructured interviews. In the case of non-response to questionnaires, a questionnaire will be resent via post a total of three times. In case of continued non-response, this will be complemented with a telephone call as a final effort for data collection.

\section{Implementation process}

- The PCS contains four items reported on a 5-point Likert scale, where a total score of 4 represents greatest self-confidence and 20 represents lowest self-confidence for managing patients with LBP. The structural validity in terms of internal consistency of the items has been shown to be good with a Cronbach's $\alpha$ coefficient $=0.73$ in a single factor model for self-confidence. ${ }^{45}$ The questionnaire has been forward-translated by our research group from English to Swedish.

- The PABS-PT consists of two factors where higher scores represent more treatment orientation regarding that factor. One factor with 10 items measures the biomedical treatment orientation (score 0-60) and one with 9 items measures the biopsychosocial treatment orientation (score 0-54). ${ }^{46}$ Each item is rated on a 6-point Likert scale ranging from $1=$ 'totally disagree' to $6=$ 'totally agree'. The internal consistency of the biomedical factor has been shown to be good with a Cronbach's $\alpha$ range of between 0.77 and 0.84. Furthermore, the biopsychosocial factor has been shown to be adequate with a Cronbach's $\alpha$ range of between 0.62 and $0.68 .{ }^{65}$ Construct validity and responsiveness to educational interventions have been shown to be positive along with the test-retest reliability with reported intraclass correlation coefficient (ICC) on the biomedical factor of 0.81 and on the biopsychosocial factor of $0.65 .{ }^{65}$ The questionnaire has been forward-translated from English to Swedish in a previously published study. ${ }^{66}$

- The Determinants of Implementation Behaviour Questionnaire (DIBQ) was originally constructed based on the domains of the TDF. ${ }^{41}{ }^{67}$ Confirmatory factor analysis resulted in a modified 93-item questionnaire assessing 18 domains with sufficient discriminant validity. Internal consistency of the items for the 18 domains was good, ranging from 0.68 to 0.93 for the Cronbach's $\alpha$ coefficient. ${ }^{68}$ The questionnaire has been forward-translated by our research group from English to Swedish. After face validity consensus in our research group regarding relevant domains for the implementation of BetterBack $\odot$ MOC, the questionnaire was shortened to the following domains: knowledge, skills, beliefs about capabilities, beliefs about consequences, intentions, innovation, organisation, patient, social influence and behavioural regulation, totalling to 57 items. Questions were adapted to the context of HCP-reported determinants of an 'expected' implementation of BetterBack $\odot$ MOC for measurement directly after the HCP education and training workshop. HCP-reported determinants retained original wording for the questionnaires at 3 and 12 months after the implementation of BetterBack $\odot$ MOC. The response scale used for each DIBQ question in our study is a 5-point Likert scale ranging from $1=$ 'totally agree' to $5=$ 'totally disagree'.

\section{Implementation outcome measures}

- At 12 months after baseline, data will also be extracted from the public healthcare regional registry for the total number of patient visits for LBP, the number of patients needing primary care multimodal pain team treatment, the number referred to specialist 
pain clinic, orthopaedic or neurosurgical care, and the number receiving surgery.

- Clinical reasoning and process evaluation tool (CRPE tool): grade of patients' functional impairment and activity limitation according to the ICF brief core set for LBP is assessed by the physiotherapist at baseline and final clinical contact, where light, moderate, severe and very severe impairment/limitation are coded $0-4$, respectively. A total score for baseline and follow-up measures is calculated from the sum of the functional impairment divided by the number of functional impairments, and a similar total score is calculated for activity limitations. ${ }^{47} \mathrm{~A}$ worsening of functional impairments and activity limitations measured at follow-up with the CRPE will be considered in the analysis of adverse events. Swedish Classification of Health Interventions (KVÅ) codes for assessment and treatment interventions will be assessed to analyse coherence with the Swedish best practice clinical recommendations. International Classification of Diseases-10 diagnosis codes will also be recorded.

- The Keele STarT Back Screening Tool is reported by patients at baseline providing a stratification of prognostic risk of persistent pain. The overall score ranging from 0 to 9 is used to separate the low-risk patients from the medium-risk subgroups, where patients who achieve a score of 0-3 are classified into the low-risk subgroup and those with scores of 4-9 into the medium-risk subgroup. To identify the high-risk subgroup, the last five items must score 4 or $5 .^{69-71}$

- Focus groups performing qualitative Strengths, Weaknesses, Opportunities and Threats (SWOT) analyses will be conducted by HCPs between 3 and 6 months after implementation.

- Semistructured interviews with 10 HCPs at 3 months after implementation will be conducted to investigate determinants of implementation behaviour and if other determinants need to be added to the DIBQ. The interviews will be deductively analysed according to the $\mathrm{TDF}^{41}$ and $\mathrm{BCW}^{39}$ frameworks.

- Semistructured interviews investigating patient experience of receiving care for LBP will be performed on 10 patients. These patients will have received care after implementation of the BetterBack $\odot$ MOC.

- Economic costs of developing the BetterBack $\odot$ MOC as well as performing the implementation strategy (staff time, HCP training and printed resources).

\section{Patient outcome measures}

- NRS-LBP intensity during the latest week is an 11-point scale consisting of integers from 0 through 10, with 0 representing 'No pain' and 10 representing 'Worst imaginable pain'. Previous research in an LBP cohort has shown a test-retest reliability ICC of 0.61 , a common SD of 1.64 points, SE of measure of 1.02 and minimal clinically important difference (MCID) in LBP after treatment of $2 .{ }^{72} 73$
- ODI V.2.1 assesses patients' current LBP-related limitation in performing activities such as personal care, lifting, walking, sitting, standing, sleeping, sex life, social life and travelling. The ODI consists of 10 items with response scales from 0 to 5 , where higher values represent greater disability. The ODI is analysed as a 0-100 percentage variable, where lower scores represent lower levels of LBP disability. A reduction of 10 points is considered the MCID in LBP after treatment. ${ }^{50}{ }^{70}$ In Scandinavian conditions, the coefficient of variation, ICC and internal consistency of the ODI is $12 \%, 0.88-0.91$ and 0.94 , respectively. ${ }^{74-76} \mathrm{Good}$ concurrent validity has also been shown. ${ }^{75}$

- The EQ-5D measures generic health-related quality of life and is computed into a $0-1.00$ scale from worst to best possible health state by using the Swedish value sets. ${ }^{77}$ A reduction of 0.08 points is considered the MCID in LBP after treatment. ${ }^{78}$ The mean change after treatment for LBP has been reported to be 0.12 $(\mathrm{SD} \pm 0.30){ }^{79}$

- The BIPQ analyses cognitive illness representations (consequences, outcome expectancy, personal control, treatment control and knowledge), emotional representations (concern and emotions) as well as illness comprehensibility. An overall score of $0-80$ represents the degree to which the LBP is perceived as threatening or benign, where a higher score reflects a more threatening view of the illness. ${ }^{52}$ The BIPQ has been shown to be valid and reliable in a Scandinavian sample of patients with subacute and chronic LBP. The BIPQ has a Cronbach's $\alpha$ of 0.72 and a testretest ICC of 0.86, an ICC range for individual items from 0.64 to 0.88 , an SE of measurement of 0.63 and minimal detectable change of $1.75 .^{80}$

- The PEI has a score range between 0 and 12, with a higher score intended to reflect higher patient selfcare enablement. ${ }^{59}$

- PGIC asks the patient to rate the degree of change in LBP-related problems from the beginning of treatment to the present. This is measured with a balanced 11-point numerical scale. A reduction of 2 points is considered the MCID in LBP after treatment. ${ }^{60}$

- PS is measured with a single-item patient-reported question. The question asks 'Over the course of treatment for this episode of LBP or leg pain, how satisfied were you with the care provided by your health care provider? Were you very satisfied (1), somewhat satisfied (2), neither satisfied nor dissatisfied (3), somewhat dissatisfied (4), or very dissatisfied (5)?' ${ }^{61}$

- Economic costs of health service utilisation.

\section{Data management}

All paper-based questionnaire data will remain confidential and will be kept in a lockable filing cabinet in the research group office. A password-protected coded database only accessible to the research team will be kept on a data storage drive in the research department. The research team will regularly monitor the integrity of trial 
data. Trial conduct will be audited on a weekly basis by the research team.

\section{Statistical analysis}

Statistical significance will be assessed with an alpha level of 0.05 . All results will be reported as estimates of mean \pm SD and also effect size (eg, mean difference) with 95\% CIs. An intention-to-treat (ITT) principle applying multiple imputation will be used. A sensitivity analysis will compare per-protocol and ITT databases. A sensitivity analysis will also be used to assess the significance of a washout period by comparing the complete database against the same database without data collected during the 2 weeks in conjunction with the BetterBack $\odot$ implementation in each cluster.

\section{Implementation process and outcome analysis}

Analysis of variance statistics comparing baseline and follow-up means will be used for implementation process and outcome measures. Causal mediation analysis will be used to analyse indirect mediational effects of multiple putative determinants of implementation behaviour measured with the DIBQ directly after the HCP education and training workshop (intention stage) or at 3 or 12 months (volition stages) on the effect of baseline PCS or PABS-PT or 3-month or 12-month follow-up measurement of PCS or PABS-PT. If the HCP education and training workshop does not have a casual effect on improved prospective outcomes, we will analyse where the causal pathway breaks down. Causal mediation analysis will be performed using the program PROCESS ${ }^{81}$ within IBM SPSS Statistics V.23 (figure 6).

Patient outcome measures for the control and intervention groups will be compared using multilevel analyses of repeated measurements and experiment condition as fixed effects and participants and clusters as random effects with IBM SPSS. Fixed-effect interactions between the experimental condition and The Keele STarT Back Screening Tool will also be assessed. Patient population-specific MCID will be assessed for primary and secondary outcomes based on an anchor method where PGIC serves as an anchor. Applying a 1-1-1 multilevel mediation procedure with all effects random in MPLUS, the products of (1) the independent variable (experimental condition: control or intervention) to the mediator (change in BIPQ, PEI), and (2) the mediator to the dependent variable (change in NRS, ODI or secondary outcome scores pretreatment to post-treatment) when the independent variable is taken into account, will be tested for mediation (figure 7).

\section{Economic analysis}

The reference case analysis is based on a healthcare sector perspective. The EQ-5D will be used to calculate the ratio of costs to QALY saved for patients. The ICERs for the multifaceted implementation strategy and the usual care condition will be calculated and plotted on a cost-effectiveness plane. This is based on the Swedish guideline-priced direct costs of health service utilisation, organisational costs of developing the BetterBack $\odot$ MOC, as well as performing the implementation strategy and overall intervention clinical outcome effectiveness. The ICER will also be calculated per patient avoiding specialist care. To estimate a distribution of costs and health measures and CIs for ICER, bootstrapping will be used.

\section{Data monitoring}

All outcome questionnaires are formatted for use of scan processing software for automated data entry into the Statistical Package for the Social Sciences package. KS, who is not blinded to treatment allocation, will perform regular data checks during data entry and provide feedback when necessary to HCPs regarding data omissions. KS will also double-check data entry to detect and correct input errors, and range checks will be undertaken prior to data analysis.

\section{Ethics and dissemination}

The ethics application including consent forms in Swedish is available on request to the authors. There are no known risks for participants. Voluntarily participating HCPs will complete questionnaires. All participating patients are informed orally and in writing about the study on the first visit at participating primary healthcare clinics. They are informed that participation is voluntary and that they can at any time withdraw their participation. The HCP intervention will not be affected by the patient's decision to participate or not participate in the study. Data collection will not be performed for those not participating. A signed patient consent form will be collected from patients by the HCP before baseline measures are collected and intervention is commenced according to the study protocol. All collected data will be entered into a database accessible to the authors. A code list will be created where each participant will be represented by a code so that the database will be anonymous. The code list with personal data will be stored separately in locked filing cabinets at Linköping University to protect confidentiality before, during and after the study. Data analyses and reporting will be performed using the de-identified database. The authors plan to disseminate the findings through manuscript publications in scientific journals and presentation at conferences.

\section{Patient and public involvement}

The adaptation of best practice clinical guidelines to the Swedish context, the construction of the BetterBack $\odot$ MOC, as well as the development of the research question, study design and outcomes measures involved interpretation of literature and professional experience of the patient net benefit versus harms and costs. Specific investigations of priorities, experience and preferences of the patients in the Östergötland healthcare region were not performed in this development phase. No patient advisors or other public are involved in the study. HCPs 


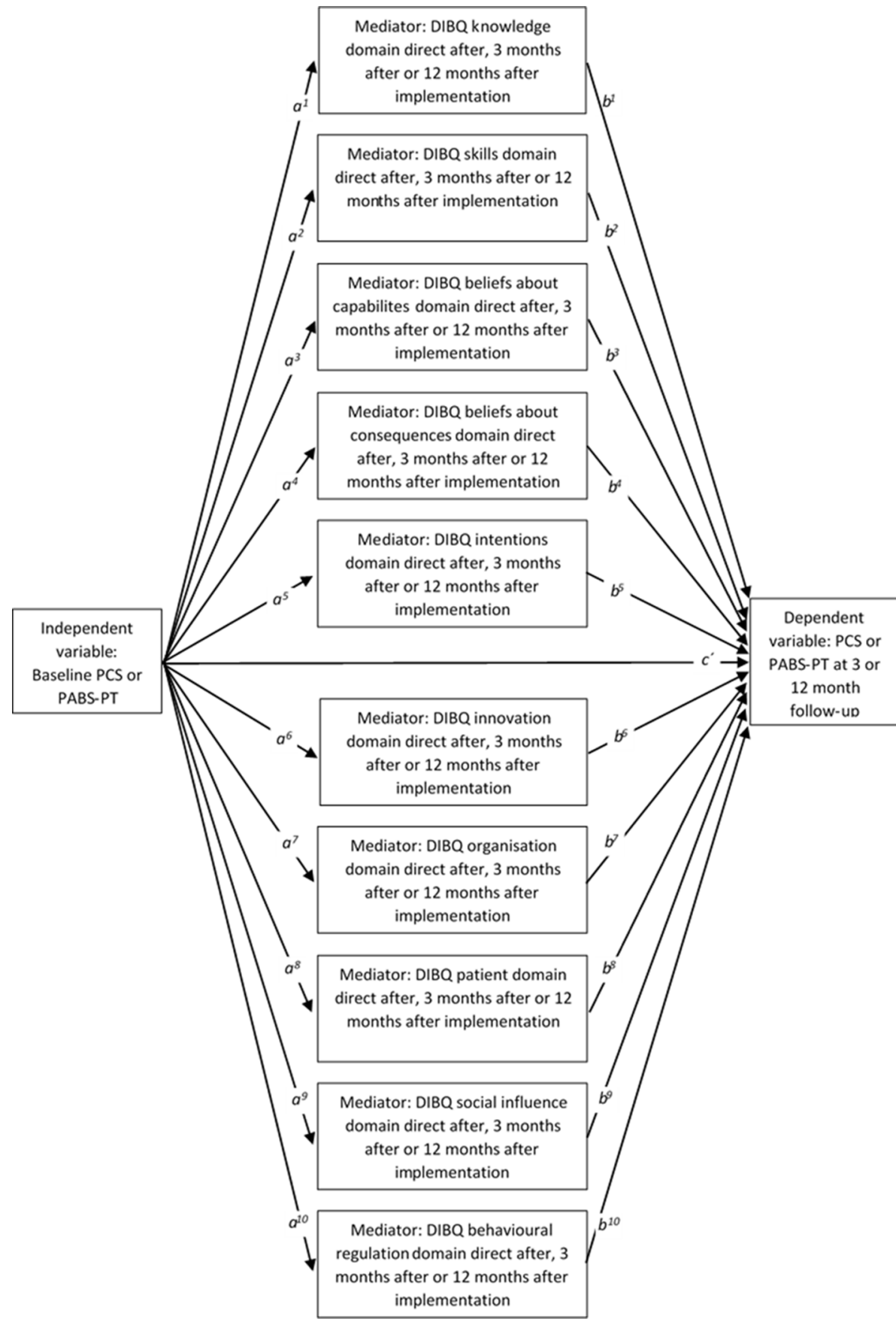

Figure 6 Causal mediation model to analyse indirect mediational effects $\left(a^{k} b^{k}\right)$ of multiple putative determinants of implementation behaviour measured with the DIBQ directly after the healthcare practitioner education/training workshop (intention stage) or at 3 or 12 months (volition stages) for the effect of baseline PCS or PABS-PT on 3-month or 12-month followup measurement of PCS or PABS-PT (c). DIBQ, Determinants of Implementation Behaviour Questionnaire; PABS-PT, Pain Attitudes and Beliefs Scale for physical therapists; PCS, Practitioner Confidence Scale.

working with patients with LBP at their clinics ask consecutive patients to participate in the study and adhere to the prescribed intervention. Patients have no other involvement in recruitment and conduct of the study. Semistructured interviews on 10 patients randomly selected will investigate the priorities, experience, burden and preferences of the intervention. Patients' satisfaction regarding the intervention is assessed by the patients themselves through a questionnaire. The dissemination of the study findings to participating patients will occur through popular science summary publication.

\section{Internal pilot trial results}

The initial implementation of the BetterBack $\odot$ MOC in cluster 1 allowed for an internal pilot to determine the HCP acceptability of the intervention and trial within the first cluster. ${ }^{32} 33$ A progression criteria for continuing to the main trial required that HCPs who have completed 


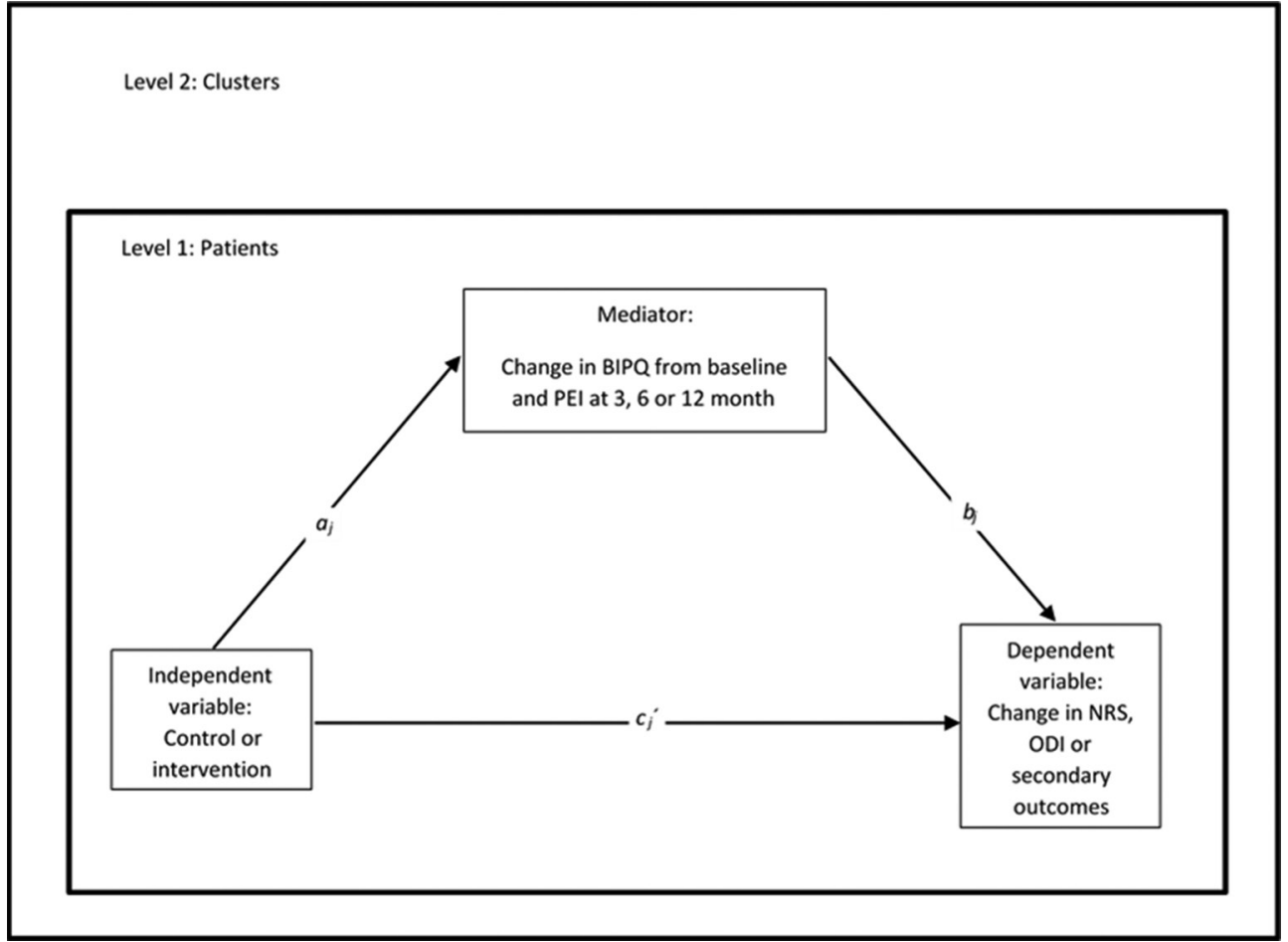

Figure 7 1-1-1 multilevel mediation model with all variables measured at level 1 , but all causal paths (direct $=c_{i}{ }^{\prime}$, indirect $=a b_{j}$ and total effects $=c_{j}{ }^{\prime}+a_{i} b_{j}$ ) are allowed to vary between level 2 clusters. BIPQ, Brief IIIness Perception Questionnaire; NRS, Numeric Rating Scale; ODI, Oswestry Disability Index; PEI, Patient Enablement Index.

the BetterBack $;$ education and training workshop rate on average a maximum of 2.5 out of 5 on the following determinants of implementation behaviour question: I expect that the application of BetterBack $\odot$ MOC will be useful $(1=$ agree completely to $5=$ do not agree at all). The 27 HCPs participating in the internal pilot in cluster 1 responded to the question with a mean value of 1.7 (SD 0.8 ), which subsequently fulfilled the HCP progression criteria.

The resulting internal pilot patient flow for April and May was $n=28$ and $n=28$ for cluster 1 west (intervention), $\mathrm{n}=5$ and $\mathrm{n}=12$ for cluster 2 central (control), as well as $\mathrm{n}=14$ and $\mathrm{n}=22$ for cluster 3 east (control) consecutively. This informed the decision to move the cluster 2 transition from control to intervention condition to occur later in the schedule, planned for September 2017, to allow for more control condition patient recruitment and data collection. The flow of patient recruitment and the process of 3-month follow-up in the internal pilot were used to inform the optimal time point of patient-reported primary outcome for the main trial. Our initial planning was to measure patient-reported primary outcome at 6 months post baseline based on the definition of persistence/chronicity of symptoms being often defined in the literature to be of 3 and up to 6 months in duration. ${ }^{82}$ Our internal pilot study had a 3-month follow rate of $80 \%$ resulting after up to three reminders sent to many of these patients. This informed of a likely risk of non-response at later follow-up time points. Furthermore, feedback from participating HCPs even reported a larger clinical interest in 3-month patient follow-up data.
Therefore the internal pilot informed the choice to revise our patient-reported primary outcomes to 3 months post baseline with subsequent amendments of the trial registration on ClinicalTrials.gov: NCT03147300.

Our internal pilot study was also used to assess baseline variation and change over 3 months in HCP and patient-reported primary outcome measures in the control and intervention arms to aid calibration of the sample size calculation. Multilevel analyses of repeated measurements and experiment condition as fixed effects and participants and clusters as random effects revealed an intracluster correlation of $<0.01$ for all primary outcomes measures. A small effect size in favour of the intervention condition was shown for HCP-reported PCS $(\mathrm{d}=0.33)$ directly after implementation but increased to a moderate effect size after 3 months $(d=0.51)$. Patient-reported NRS showed a small effect size $(\mathrm{d}=0.28)$. Therefore, the internal pilot data supported our a priori sample size calculation for the main trial regarding PCS and NRS. However no effect size difference was observed between experimental conditions for ODI. It is possible that when statistical power improves when the trial progresses, potential differences in ODI may be detectable between experimental conditions.

\section{CONCLUSION}

The effectiveness-implementation hybrid type 2 trial with dogleg stepped cluster structure allowed for the use of an internal pilot to inform feasibility and optimise method efficiency for the progression of the trial. 
Contributors $A A$ and $B O ̈$ formulated the trial's original aims and hypothesis. AA, KS and $B O ̈$ developed intervention materials. $A A, K S, P E, P N$ and $O ̈ B$ designed the study methodology. $A A, P N$ and $B O ̈$ procured funding for the trial. $A A, K S, P E, P N$ and $O B B$ have reviewed and finalised the protocol.

Funding This work was supported by the Research Council in Southeast Sweden (grant number: FORSS*660371) and the Swedish Research Council (grant number: 2017*01444).

Competing interests None declared.

Patient consent Detail has been removed from this case description/these case descriptions to ensure anonymity. The editors and reviewers have seen the detailed information available and are satisfied that the information backs up the case the authors are making.

Ethics approval Ethical clearance for the study (Dnr: 2017-35/31) has been attained through the Regional Ethics Committee in Linköping.

Provenance and peer review Not commissioned; externally peer reviewed.

Open Access This is an Open Access article distributed in accordance with the Creative Commons Attribution Non Commercial (CC BY-NC 4.0) license, which permits others to distribute, remix, adapt, build upon this work non-commercially, and license their derivative works on different terms, provided the original work is properly cited and the use is non-commercial. See: http://creativecommons.org/ licenses/by-nc/4.0/

(C) Article author(s) (or their employer(s) unless otherwise stated in the text of the article) 2018. All rights reserved. No commercial use is permitted unless otherwise expressly granted.

\section{REFERENCES}

1. Hoy D, Bain C, Williams $G$, et al. A systematic review of the global prevalence of low back pain. Arthritis \& Rheumatism 2012;64:2028-37.

2. Hoy D, March L, Brooks P, et al. The global burden of low back pain: estimates from the Global Burden of Disease 2010 study. Ann Rheum Dis 2014;73:968-74.

3. Dionne CE, Dunn KM, Croft PR, et al. A consensus approach toward the standardization of back pain definitions for use in prevalence studies. Spine 2008;33:95-103.

4. Smart KM, O'Connell NE, Doody C. Towards a mechanisms-based classification of pain in musculoskeletal physiotherapy? Physical Therapy Reviews 2008;13:1-10.

5. Williams CM, Henschke N, Maher CG, et al. Red flags to screen for vertebral fracture in patients presenting with low-back pain. Cochrane Database Syst Rev 2013;1:CD008643.

6. Henschke N, Maher CG, Ostelo RW, et al. Red flags to screen for malignancy in patients with low back pain. Cochrane Database Syst Rev 2013;2:CD008686.

7. Konstantinou K, Dunn KM. Sciatica: review of epidemiological studies and prevalence estimates. Spine 2008;33:2464-72.

8. Yabuki S, Otani K, Sekiguchi M, Fukumori N, Takegami M, et al. Prevalence of lumbar spinal stenosis, using the diagnostic support tool, and correlated factors in Japan: a population-based study. Journal of Orthopaedic Science 2013;18:893-900.

9. Brinjikji W, Luetmer PH, Comstock B, et al. Systematic literature review of imaging features of spinal degeneration in asymptomatic populations. Am J Neuroradiol 2015;36:811-6.

10. Wåhlin C, Ekberg K, Persson J, et al. Association between clinical and work-related interventions and return-to-work for patients with musculoskeletal or mental disorders. J Rehabil Med 2012;44:355-62.

11. Nilsing E, Söderberg E, Öberg B. Sickness certificates in Sweden: did the new guidelines improve their quality? BMC Public Health 2012;12:907.

12. Bernhardsson S, Öberg B, Johansson $\mathrm{K}$, et al. Clinical practice in line with evidence? A survey among primary care physiotherapists in western Sweden. J Eval Clin Pract 2015;21:1169-77.

13. Sundhedsstyrelsen. The national clinical guideline for non-surgical treatment of recently lumbar nervous system impact (lumbar radiculopathy) provides recommendations on non-surgical treatment options. 2016 https://sundhedsstyrelsen.dk/da/udgivelser/2016/ lumbal-nerverodspaavirkning-ikke-kirurgisk-behandling (accessed 3 May 2016).

14. Sundhedsstyrelsen. National clinical guidelines for non-surgical treatment of newly occurring lower back pain. $2016 \mathrm{https}: / /$ sundhedsstyrelsen.dk/da/udgivelser/2016/nkr-laenderygsmerter (accessed 3 May 2016).
15. National Clinical Guideline Centre (NICE). Low back pain and sciatica in over 16s: assessment and management. $2016 \mathrm{https} / / / \mathrm{www}$. nice.org.uk/guidance/indevelopment/gid-cgwave0681/documents (accessed 3 May 2016).

16. Thorstensson CA, Garellick G, Rystedt H, et al. Better management of patients with osteoarthritis: development and nationwide implementation of an evidence-based supported osteoarthritis selfmanagement programme. Musculoskeletal Care 2015;13:67-75.

17. Skou ST, Roos EM. Good Life with osteoArthritis in Denmark (GLA:DTM): evidence-based education and supervised neuromuscular exercise delivered by certified physiotherapists nationwide. BMC Musculoskelet Disord 2017;18:72.

18. Thorstensson C, Dahlberg L, Garellick G. The BOA-register annual report. 2014 https://boa.registercentrum.se (accessed 03 May 2016).

19. Skou ST, Roos EM. GLA:D annual report. 2015 www.glaid.dk (accessed 3 May 2016).

20. Briggs AM, Jordan JE, Jennings $M$, et al. A framework to evaluate musculoskeletal models of care. Cornwall: Global Alliance for Musculoskeletal Health of the Bone and Joint Decade, 2016.

21. Fixsen DL, Naoom SF, Blase KA, et al. Implementation research: a synthesis of the literature. Tampa, FL: University of South Florida, Louis de la Parte Florida Mental Health Institute, 2005.

22. Nilsen P. Making sense of implementation theories, models and frameworks. Implementation Science 2015;10:53.

23. Nilsen P. (red) Implementering av evidensbaserad praktik. Malmö: Gleerups, 2014.

24. Nutley SM, Walter I, Davies HTO, et al. How research can inform public services. Bristol: Policy Press, 2007.

25. Eccles MP, Mittman BS. Welcome to Implementation Science. Implement Sci 2006:1:1.

26. Suman A, Dikkers MF, Schaafsma FG, et al. Effectiveness of multifaceted implementation strategies for the implementation of back and neck pain guidelines in health care: a systematic review. Implementation Science 2015;11:126

27. Mesner SA, Foster NE, French SD. Implementation interventions to improve the management of non-specific low back pain: a systematic review. BMC Musculoskelet Disord 2016;17:258.

28. Chan A-W, Tetzlaff JM, Gotzsche PC, et al. SPIRIT 2013 explanation and elaboration: guidance for protocols of clinical trials. BMJ 2013;346:e7586.

29. Curran GM, Bauer M, Mittman B, et al. Effectiveness-implementation hybrid designs: combining elements of clinical effectiveness and implementation research to enhance public health impact. Med Care 2012;50:217-26.

30. Hooper R, Bourke L. Cluster randomised trials with repeated cross sections: alternatives to parallel group designs. BMJ 2015;350:h2925.

31. Girling AJ, Hemming K. Statistical efficiency and optimal design for stepped cluster studies under linear mixed effects models. Stat Med 2016;35:2149-66.

32. Eldridge S, Kerry S. A practical guide to cluster randomised trials in health service research. 2nd edn: Wiley \& Sons, 2012.

33. Avery KNL, Williamson PR, Gamble C, et al. Informing efficient randomised controlled trials: exploration of challenges in developing progression criteria for internal pilot studies. BMJ Open 2017;7:e013537.

34. Hoffmann TC, Glasziou PP, Boutron I, et al. Better reporting of interventions: template for intervention description and replication (TIDieR) checklist and guide. BMJ 2014;348:g1687.

35. SBU. Acute neck and back pain: preventive interventions - Effects of physical training, manual treatment and cognitive behavioral interventions. Stockholm: Swedish Agency for Health Technology Assessment and Assessment of Social Services (SBU), 2016. http:// www.sbu.se/en/publications/sbu-assesses/acute-neck-and-backpain-preventive-interventions-effects-of-physical-training-manualtreatment-and-cognitive-behavioral-interventions/.

36. The Swedish National Board of Health and Welfare. National guidelines - Methods description. https://www.socialstyrelsen.se/ SiteCollectionDocuments/metodbeskrivning-nationella-riktlinjer.pdf (accessed 3 May 2016).

37. Atkins D, Best D, Briss PA, et al. Grading quality of evidence and strength of recommendations. BMJ 2004;328:1490.

38. Slade SC, Kent P, Patel S, et al. Barriers to primary care clinician adherence to clinical guidelines for the management of low back pain: a systematic review and metasynthesis of qualitative studies. Clin J Pain 2016;32:38.

39. Michie S, van Stralen MM, West R. The behaviour change wheel: A new method for characterising and designing behaviour change interventions. Implementation Science 2011;6:42.

40. Michie S, Wood CE, Johnston M, et al. Behaviour change techniques: the development and evaluation of a taxonomic method 
for reporting and describing behaviour change interventions (a suite of five studies involving consensus methods, randomised controlled trials and analysis of qualitative data). Health Technol Assess 2015;19:1-188.

41. Cane J, O'Connor D, Michie S. Validation of the theoretical domains framework for use in behaviour change and implementation research. Implementation Science 2012;7:37.

42. Leventhal H, Phillips LA, Burns $E$. The common-sense model of self-regulation (CSM): a dynamic framework for understanding illness self-management. J Behav Med 2016;39:935-46.

43. Jack K, McLean SM, Moffett JK, Klaber Moffett J, et al. Barriers to treatment adherence in physiotherapy outpatient clinics: A systematic review. Man Ther 2010;15:220-8.

44. Michie S, Johnston M, Francis J, et al. From theory to intervention: mapping theoretically derived behavioural determinants to behaviour change techniques. Appl Psychol 2008;57:660-80.

45. Smucker DR, et al. Practitioner self-confidence and patient outcomes in acute low back pain. Arch Fam Med 1998;7:223-8.

46. Ostelo RWJG, Stomp-van den Berg SGM, Vlaeyen JWS, et al. Health care provider's attitudes and beliefs towards chronic low back pain: the development of a questionnaire. Man Ther 2003;8:214-22.

47. Cieza A, Weigl M, Disler P, et al. ICF core sets for low back pain. $J$ Rehabil Med 2004;36:69-74.

48. Jensen MP, Turner JA, Romano JM, et al. Comparative reliability and validity of chronic pain intensity measures. Pain 1999;83:157-62.

49. Chiarotto A, Deyo RA, Terwee CB, et al. Core outcome domains for clinical trials in non-specific low back pain. European Spine Journal 2015;24:1127-42.

50. Clement RC, Welander A, Stowell C, et al. A proposed set of metrics for standardized outcome reporting in the management of low back pain. Acta Orthop 2015:86:523-33.

51. Fairbank JCT, Pynsent PB. The oswestry disability index. Spine 2000;25:2940-53.

52. EuroQol Group. EuroQol-a new facility for the measurement of health related quality of life. Health Policy 1990;16:199-208.

53. Broadbent E, Petrie KJ, Main J, et al. The brief illness perception questionnaire. J Psychosom Res 2006;60:631-7.

54. Foster NE, Bishop A, Thomas E, et al. Illness perceptions of low back pain patients in primary care: what are they, do they change and are they associated with outcome? Pain 2008;136:177-87.

55. Foster NE, Thomas E, Bishop A, et al. Distinctiveness of psychological obstacles to recovery in low back pain patients in primary care. Pain 2010;148:398-406.

56. Glattacker M, Heyduck K, Meffert C. Illness beliefs and treatment beliefs as predictors of short-term and medium-term outcome in chronic back pain. J Rehabil Med 2013;45:268-76.

57. Campbell P, Foster NE, Thomas E, et al. Prognostic indicators of low back pain in primary care: five-year prospective study. The Journal of Pain 2013;14:873-83.

58. Løchting I, Garratt AM, Storheim K, et al. The impact of psychological factors on condition-specific, generic and individualized patient reported outcomes in low back pain. Health Qual Life Outcomes 2017;15:40.

59. Rööst M, Zielinski A, Petersson C, et al. Reliability and applicability of the patient enablement instrument (PEI) in a Swedish general practice setting. BMC Fam Pract 2015;16:31.

60. Kamper SJ, Maher CG, Mackay G. Global rating of change scales: a review of strengths and weaknesses and considerations for design. Journal of Manual \& Manipulative Therapy 2009;17:163-70.

61. Butler RJ, Johnson WG. Satisfaction with low back pain care. The Spine Journal 2008;8:510-21.

62. Slater H, Davies SJ, Parsons R, et al. A policy-into-practice intervention to increase the uptake of evidence-based management of low back pain in primary care: a prospective cohort study. PLoS One 2012;7:e38037.
63. Tzortziou Brown V, Underwood M, Mohamed N, et al. Professional interventions for general practitioners on the management of musculoskeletal conditions. Cochrane Database Syst Rev 2016;6:CD007495.

64. Campbell MK, Piaggio G, Elbourne DR, et al. Consort 2010 statement: extension to cluster randomised trials. $B M J$ 2012;345:e5661.

65. Mutsaers J-HAM, Peters R, Pool-Goudzwaard AL, et al. Psychometric properties of the Pain Attitudes and Beliefs Scale for Physiotherapists: A systematic review. Man Ther 2012;17:213-8.

66. Overmeer T, Boersma K, Main CJ, et al. Do physical therapists change their beliefs, attitudes, knowledge, skills and behaviour after a biopsychosocially orientated university course? J Eval Clin Pract 2009;15:724-32.

67. Huijg JM, Gebhardt WA, Crone MR, et al. Discriminant content validity of a theoretical domains framework questionnaire for use in implementation research. Implementation Science 2014;9:11.

68. Huijg JM, Gebhardt WA, Dusseldorp E, et al. Measuring determinants of implementation behavior: psychometric properties of a questionnaire based on the theoretical domains framework. Implementation Science 2014;9:33.

69. Hill JC, Dunn KM, Lewis M, et al. A primary care back pain screening tool: Identifying patient subgroups for initial treatment. Arthritis \& Rheumatism 2008;59:632-41.

70. Hill JC, Vohora K, Dunn KM, et al. Comparing the start back screening tool's subgroup allocation of individual patients with that of independent clinical experts. Clin J Pain 2010;26:783-7.

71. Hill JC, Dunn KM, Main CJ, et al. Subgrouping low back pain: a comparison of the start back tool with the örebro musculoskeletal pain screening questionnaire. European Journal of Pain 2010;14:83-9.

72. Childs JD, Piva SR, Fritz JM. Responsiveness of the numeric pain rating scale in patients with low back pain. Spine 2005;30:1331-4.

73. Ostelo RW, Deyo RA, Stratford P, et al. Interpreting change scores for pain and functional status in low back pain: towards international consensus regarding minimal important change. Spine 2008;33:90-4

74. Grotle M, Brox JI, Vøllestad NK. Cross-cultural adaptation of the norwegian versions of the roland-morris disability questionnaire and the oswestry disability index. J Rehabil Med 2003;35:241-7.

75. Lauridsen HH, Hartvigsen J, Manniche C, et al. Danish version of the Oswestry Disability Index for patients with low back pain. Part 1: Cross-cultural adaptation, reliability and validity in two different populations. Europ Spine J 2006;15:1705-16.

76. Lauridsen $\mathrm{HH}$, Hartvigsen J, Manniche $\mathrm{C}$, et al. Danish version of the Oswestry disability index for patients with low back pain. Part 2: Sensitivity, specificity and clinically significant improvement in two low back pain populations. Europ Spine J 2006;15:1717-28.

77. Burström K, Sun S, Gerdtham U-G, et al. Swedish experience-based value sets for EQ-5D health states. Quality Life Res 2014;23:431-42.

78. Walters SJ, Brazier JE. Comparison of the minimally important difference for two health state utility measures: EQ-5D and SF-6D. Quality of Life Research 2005;14:1523-32.

79. Soer R, Reneman MF, Speijer BLGN, et al. Clinimetric properties of the EuroQol-5D in patients with chronic low back pain. The Spine Journal 2012;12:1035-9.

80. Loechting I, Garratt AM, Storheim K, et al. Evaluation of the brief illness perception questionnaire in sub-acute and chronic low back pain patients: data quality, reliability and validity. J Pain Relief 2013;2:122.

81. Hayes AF. PROCESS: A versatile computational tool for observed variable mediation, moderation, and conditional process modeling [White paper]. 2012 http://www.afhayes.com/public/process2012. pdf.

82. Merskey H, Bogduk N. Classification of chronic pain. 2nd ed. Seattle: IASP Press, 1994:1. 